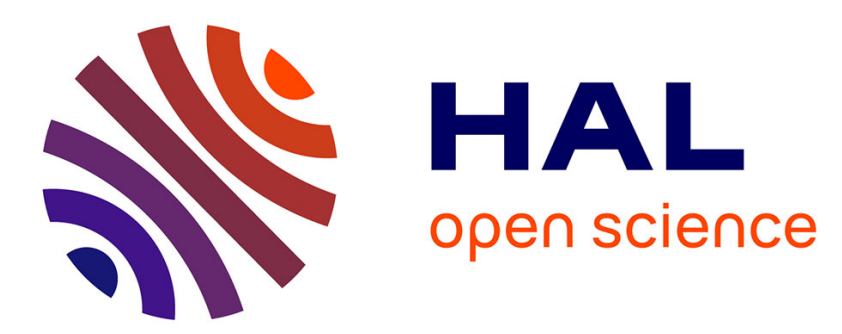

\title{
Streamer-induced transport in the presence of trapped ion modes in tokamak plasmas
}

\author{
A. Ghizzo, D. del Sarto, X. Garbet, Y. Sarazin
}

\section{To cite this version:}

A. Ghizzo, D. del Sarto, X. Garbet, Y. Sarazin. Streamer-induced transport in the presence of trapped ion modes in tokamak plasmas. Physics of Plasmas, 2010, 17 (9), pp.092501. 10.1063/1.3474955 . hal-01791697

\section{HAL Id: hal-01791697 \\ https://hal.univ-lorraine.fr/hal-01791697}

Submitted on 14 May 2018

HAL is a multi-disciplinary open access archive for the deposit and dissemination of scientific research documents, whether they are published or not. The documents may come from teaching and research institutions in France or abroad, or from public or private research centers.
L'archive ouverte pluridisciplinaire HAL, est destinée au dépôt et à la diffusion de documents scientifiques de niveau recherche, publiés ou non, émanant des établissements d'enseignement et de recherche français ou étrangers, des laboratoires publics ou privés. 


\section{Streamer-induced transport in the presence of trapped ion modes in tokamak plasmas}

A. Ghizzo, D. Del Sarto, X. Garbet, and Y. Sarazin

Citation: Physics of Plasmas 17, 092501 (2010); doi: 10.1063/1.3474955

View online: https://doi.org/10.1063/1.3474955

View Table of Contents: http://aip.scitation.org/toc/php/17/9

Published by the American Institute of Physics

\section{Articles you may be interested in}

Effects of $\mathrm{E} \times \mathrm{B}$ velocity shear and magnetic shear on turbulence and transport in magnetic confinement devices Physics of Plasmas 4, 1499 (1997); 10.1063/1.872367

Shear-flow trapped-ion-mode interaction revisited. I. Influence of low-frequency zonal flow on ion-temperaturegradient driven turbulence

Physics of Plasmas 22, 082303 (2015); 10.1063/1.4928102

Global gyrokinetic simulations of trapped-electron mode and trapped-ion mode microturbulence

Physics of Plasmas 22, 082302 (2015); 10.1063/1.4927920

Physics of zonal flows

Physics of Plasmas 13, 055502 (2006); 10.1063/1.2178779

Electron temperature gradient driven turbulence

Physics of Plasmas 7, 1904 (2000); 10.1063/1.874014

Influence of sheared poloidal rotation on edge turbulence

Physics of Fluids B: Plasma Physics 2, 1 (1990); 10.1063/1.859529

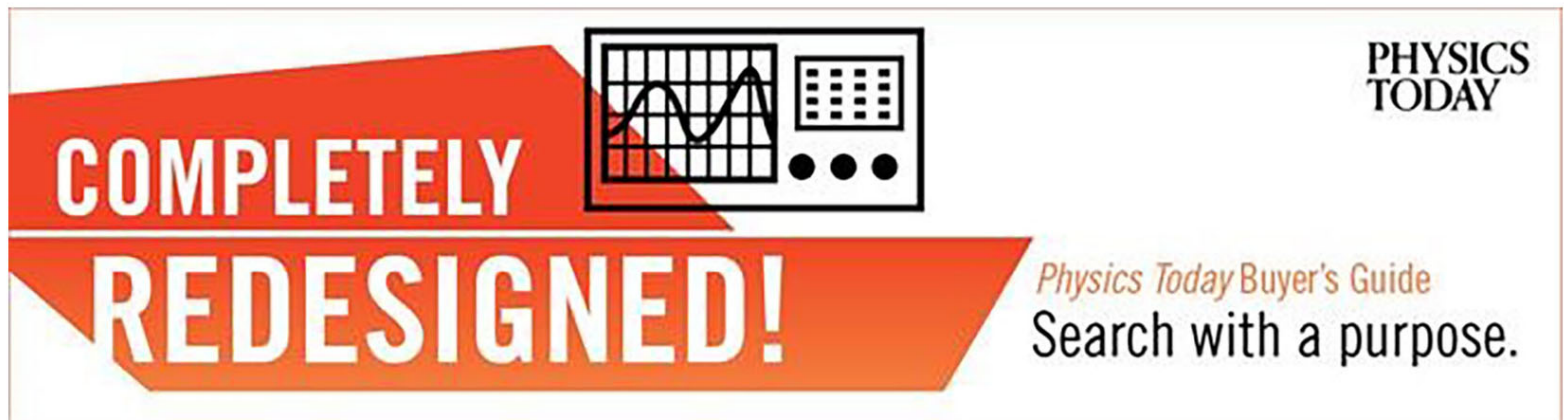




\title{
Streamer-induced transport in the presence of trapped ion modes in tokamak plasmas
}

\author{
A. Ghizzo, ${ }^{1}$ D. Del Sarto, ${ }^{1}$ X. Garbet, ${ }^{2}$ and Y. Sarazin ${ }^{2}$ \\ ${ }^{1}$ IJL UMR 7163, Nancy-Universités EPCS, BP 239, F-54506 Vandoeuvre les Nancy, France \\ ${ }^{2}$ IRFM, CEA Cadarache, 13108 St. Paul-Les-Durance, France
}

(Received 6 May 2010; accepted 13 July 2010; published online 1 September 2010)

\begin{abstract}
Global gyrokinetic Vlasov simulations for trapped ion modes are performed by solving a Vlasov equation averaged over the cyclotron and bounce motions of trapped ions. The distribution function, for trapped ions, is then calculated in a two-dimensional phase space, parametrized by the longitudinal action (energy) and the magnetic moment in presence of magnetic shear. The physical mechanism of the saturation processes between streamerlike structures and zonal flows in relation to the suppression of turbulent transport is discussed. The magnetic shear is identified to play a key role in the dominant streamer-induced transport regime, which exhibits a Bohm-like scaling. The interaction of streamerlike structures with plasma turbulence is shown to produce the inverse cascade that condenses onto long-wavelength trapped ion structures, on the basis on wave triad interactions. () 2010 American Institute of Physics. [doi:10.1063/1.3474955]
\end{abstract}

\section{INTRODUCTION}

Plasma turbulence, which is believed to be one of the key components which determines the plasma behavior, is often characterized by the formation of anisotropic structures such as zonal flows or streamer. ${ }^{1-3}$ In the context of tokamak plasmas, zonal flow is $n=0$ electrostatic potential fluctuations, driven by nonlinear interactions, which transfer energy from the finite $n$-drift waves to the $n=0$ flow, with $n$ being the toroidal number here. Thus zonal flows are elongated asymmetric purely radial variations of the electrostatic potential, whereas streamers are convective cells localized in the potential direction and extended radially, which is the opposite limit of anisotropy from that of the zonal flows. An important role of zonal flow in regulating turbulence and transport is now broadly accepted. ${ }^{4,5}$ Indeed zonal flow may regulate and partially suppress drift-wave turbulence and transport. Zonal flow dynamics are mesoscopic phenomena occurring on spatial scales between those of the turbulence correlation length and characteristic scale lengths of the density profiles.

On the other hand, it can be expected that the presence of streamers may have a significant impact on the resulting turbulent transport. On general grounds, it is reasonable to expect an enhancement of turbulent transport in presence of streamers. Such structures may occur in the saturation processes in ion temperature gradient (ITG) instabilities and, in particular, in the case of the growth of trapped ion modes (TIMs) in the collisionless regime. Usually both structures, zonal flows, or streamers do not occur simultaneously and the appearance of streamers may be usually an indication of the relative weakness of zonal flow generation in plasmas. However the saturation mechanism of fully developed collisionless trapped electron mode (TEM) turbulence driven electron temperature gradient (ETG) instability is today an open question. Streamers are recently expected to occur also in the saturation process of TEMs and in ETG driven turbulence. While the generation of zonal flows in collisionless
TEM turbulence is predicted by theoretical calculations, ${ }^{6,7}$ it has been proposed recently ${ }^{8}$ that the nonlinear saturation mechanism of ETG driven TEM is not zonal flow generation (in contrast to the ITG case), but perpendicular particle diffusion, induced by $\mathbf{E} \times \mathbf{B}$ nonlinearities on the longwavelength transport dominated modes. Competition between streamers and zonal flow has been studied in Ref. 9 for ETG and in Ref. 10 using the modulational stability method. A transport enhancement by streamers was found in Ref. 11 for ETG.

An essential and interesting question is what physical parameters determine the formation of zonal flows or streamers in ITG or ETG turbulence. In particular, trapped modes seem to play a major role in anomalous transport. In the present paper, we address the question how streamers can give rise to an increase of the transport induced by TIM instabilities. TIMs are characterized by frequencies of the order of the trapped particle precession frequency and radial scales of the order of several banana widths. These properties make TIMs have a global nature, i.e., large radial correlation lengths that can vary on the equilibrium scale length rather than on the ion gyroradius scale length usually associated with the kinetic microinstabilities. Such trapped ion driven modes are thus a simple prototype of kinetic instability since they are driven through the resonant interaction between a wave and trapped ions through their precession motion. They imply low toroidal numbers $(n<100)$ and lead to streamerinduced turbulence.

Major mechanisms of microturbulence transport are studied here: forward cascade processes and inverse cascade (which behaves like a critical nonlinear phenomenon for kinetic instabilities near threshold), polarization effect on saturation, turbulence stabilization by zonal flow, or reversed magnetic shear. The streamers are mostly destroyed by the zonal flow generation, which is found to be important in the stabilization by reversed magnetic shear. Nonlinear threewave coupling is occurring in TIM instability and long- 
wavelength streamerlike structures are observed in simulation as the result of a condensation process in the saturation mechanism. Although here, our focus is on the dynamics of TIM in the context of ITG turbulence, our model may also be extended to other types of streamer-dominated turbulence, driven for instance by TEM.

\section{THE KINETIC MODEL FOR TIM}

\section{A. The gyrokinetic Vlasov equation}

In order to provide a simple physical picture of the bounce motion and TIMs, we review the guiding center treatment of trapped ions in high aspect ratio $(\varepsilon \ll 1)$ tokamaks given by Kadomtsev and Pogutse. ${ }^{12}$ The motion of a charge particle in a tokamak is described by the motion of the guiding center after averaging the cyclotron motion. It is then characterized by three invariants: the adiabatic invariant $\mu$, the energy $E$, and the toroidal kinetic momentum $M$. Their expressions are

$$
\begin{aligned}
& \mu=\frac{m_{i} v_{\perp}^{2}}{2 B\left(\mathbf{x}_{G}\right)}, \\
& E=\frac{1}{2} m_{i} v_{G \|}^{2}+\mu B\left(\mathbf{x}_{G}\right), \\
& M=e \psi\left(\mathbf{x}_{G}\right)+m_{i} R\left(\mathbf{x}_{G}\right) v_{G \varphi},
\end{aligned}
$$

where $e$ and $m_{i}$ are the charge and mass of trapped ions, and

$$
B(r, \theta)=b(r, \theta) B_{0}=B_{0}\left(1+\frac{r}{R_{0}}(1-\cos \theta)\right)
$$

is the magnetic field modulus for large aspect ratio tokamak, $R(r, \theta)$ is the major radius, and $\psi(r)$ is the poloidal flux normalized to $2 \pi . B_{0}$ is the minimal value of $B$ at $\theta=0\left(R=R_{0}\right.$ being then the major radius). Here the label $G$ refers to the guiding center and $\mathbf{x}_{G}$ corresponds to $(r, \theta)$ coordinates.

The initial work of Kadomtsev and Pogutse indicated that the TIM has a real frequency below the diamagnetic drift frequency $\omega_{*}$ and below the bounce frequency $\omega_{b}$. TIMs were obtained by averaging over the fast scales (cyclotron and bounce motions). This task is made easier in the framework of the Hamiltonian formalism, ${ }^{13-17}$ when the phase space $(\mathbf{x}, \mathbf{p})$ may be described by a system of three action variables $J_{k}(\mathbf{x}, \mathbf{p})(k=1,2,3)$ and their three corresponding angular variables $\phi_{k}(\mathbf{x}, \mathbf{p})$. Foundations of nonlinear gyrokinetic theory may be found in Refs. 18 and 19.

Such canonically conjugate variables $J_{k}$, constants of motion, and $\phi_{k}$ varying linearly with time, result from the integrality of the trajectories in the unperturbed tokamak magnetic configuration. Thus the invariants $E, \mu$, and $M$ are related to the action variables $J_{1}, J_{2}$, and $J_{3}$ when we assumed that the Hamiltonian at equilibrium is a function of only $\mathbf{J}=\left(J_{1}, J_{2}, J_{3}\right)$, i.e., $H=H_{0}(\mathbf{J})$ and the laws of motion are

$$
\frac{d \mathbf{J}}{d t}=-\frac{\partial H_{0}(\mathbf{J})}{\partial \phi}=0,
$$

$$
\frac{d \phi}{d t}=\frac{\partial H_{0}(\mathbf{J})}{\partial \mathbf{J}}=\boldsymbol{\Omega}(\mathbf{J})=\left(\omega_{c}, \omega_{b}, \omega_{d}\right),
$$

or equivalently

$$
J_{k}=\text { const } \quad \text { and } \quad \phi_{k}=\Omega_{k} t+\text { const. }
$$

The trajectory can be rewritten as (see Ref. 16)

$$
\begin{aligned}
& \theta_{G}=\hat{\theta}\left(\boldsymbol{J}, \phi_{2}\right), \\
& \varphi_{G}=\phi_{3}+q\left(r_{0}\right) \hat{\theta}\left(\boldsymbol{J}, \phi_{2}\right)+\hat{\varphi}\left(\boldsymbol{J}, \phi_{2}\right), \\
& r_{G}=r_{0}+\hat{r}\left(\boldsymbol{J}, \phi_{2}\right) .
\end{aligned}
$$

The functions $\hat{\theta}\left(\boldsymbol{J}, \phi_{2}\right)$ and $\hat{\varphi}\left(\boldsymbol{J}, \phi_{2}\right)$ are periodic in $\phi_{2}$ and represent the bounce motion and the deviation from the regular precession motion, respectively. The low-frequency response for TIM is obtained by making a phase-angle average over the cyclotron phase $\phi_{1}$ and over the bounce motion phase $\phi_{2}$ (the "banana" orbit). Only the precession motion is explicitly taken into account in the model and this phaseangle average leads to [where the angular variable is $\boldsymbol{\phi}$ $\left.=\left(\phi_{1}, \phi_{2}, \phi_{3}\right)\right]$

$$
\begin{aligned}
& \omega_{k}=\frac{d \phi_{k}}{d t}=\frac{\partial \tilde{H}}{\partial J_{k}}, \\
& \left(\frac{d J_{k}}{d t}\right)_{k=1,2}=0 \quad \text { and } \quad \frac{d J_{3}}{d t} \neq 0 .
\end{aligned}
$$

Thus each adiabatic invariant reduces the dimensionality by a factor of 2 . Finally the two important variables are the precession phase $\phi_{3}=\varphi-q \theta$ [assuming in first approximation that $\hat{\varphi}=0$ in Eq. (9)] and the poloidal flux $\psi$ related to $J_{3}$ $\left[M=e \psi\left(r_{0}\right)\right.$, where $r_{0}$ is an average radius], which plays the role of the radial coordinate. In that case, $\left(\psi, \phi_{3}\right)$ space looks like the usual $(r, \theta)$ space.

The rigorous derivation of the bounce-kinetic Vlasov equation follows the Hamiltonian formalism described by Garbet et al. ${ }^{17}$ The evolution of an ensemble of particles is then described by a distribution function $f(\boldsymbol{J}, \boldsymbol{\phi}, t)$, which satisfies the Vlasov equation

$$
\frac{d f}{d t}=\frac{\partial f}{\partial t}+[f, H]=0,
$$

where

$$
\begin{aligned}
{[f, H]=} & \frac{\partial f}{\partial \phi_{1}} \frac{\partial H}{\partial J_{1}}+\frac{\partial f}{\partial \phi_{2}} \frac{\partial H}{\partial J_{2}}+\frac{\partial f}{\partial \phi_{3}} \frac{\partial H}{\partial J_{3}}-\frac{\partial f}{\partial J_{1}} \frac{\partial H}{\partial \phi_{1}} \\
& -\frac{\partial f}{\partial J_{2}} \frac{\partial H}{\partial \phi_{2}}-\frac{\partial f}{\partial J_{3}} \frac{\partial H}{\partial \phi_{3}}
\end{aligned}
$$

is the usual Poisson bracket. The introduction of a Hamiltonian $H(\boldsymbol{\phi}, \mathbf{J}, t)$ and the distribution function $f(\boldsymbol{\phi}, \mathbf{J}, t)$ in the form (i.e., as the sum of the equilibrium term and a perturbation term)

$$
H(\boldsymbol{\phi}, \mathbf{J}, t)=H_{0}(\mathbf{J})+\sum_{\mathbf{n}, \omega} \widetilde{H}_{\mathbf{n}, \omega}(\mathbf{J}) e^{i(\boldsymbol{n} \cdot \boldsymbol{\phi}-\omega t)},
$$




$$
f(\boldsymbol{\phi}, \mathbf{J}, t)=F_{0}(\mathbf{J})+\sum_{\mathbf{n}, \omega} \widetilde{f}_{\mathbf{n}, \omega}(\mathbf{J}) e^{i(\boldsymbol{n} \cdot \boldsymbol{\phi}-\omega t)}
$$

allows us to write the perturbed term $\tilde{f}_{\mathbf{n}, \omega}(\mathbf{J})$ in the form of a sum of a adiabatic term [first term in Eq. (17) and a resonant wave-particle contribution (Landau-type), where for TIM, the term $\mathbf{n} . \boldsymbol{\Omega}$ reduces to $\left.n_{3} \omega_{d}(\kappa ; s)\right]$,

$$
\begin{aligned}
\tilde{f}_{\mathbf{n}, \omega}(\mathbf{J})= & -\frac{F_{0}(\mathbf{J})}{T_{0}\left(J_{3}\right)} \tilde{H}_{\mathbf{n}, \omega}(\mathbf{J}) \\
& +\frac{F_{0}(\mathbf{J})}{T_{0}\left(J_{3}\right)} \frac{\omega-\mathbf{n} \cdot \boldsymbol{\omega}_{*}}{\omega-\boldsymbol{n} \cdot \mathbf{\Omega}(\mathbf{J})+i 0^{+}} \tilde{H}_{\mathbf{n}, \omega}(\mathbf{J}) .
\end{aligned}
$$

Here $\mathbf{n}$ denotes a triplet of integers. We have also assumed that each population (or class of solutions) is in thermal equilibrium on each magnetic surface. We can use a local description in the form

$$
F_{0}(\mathbf{J})=\frac{n_{0}\left(J_{3}, t\right)}{\left[2 \pi m_{i} T_{0}\left(J_{3}\right)\right]^{3 / 2}} \exp \left[-\frac{H_{0}(\mathbf{J})}{T_{0}\left(J_{3}\right)}\right],
$$

where the density $n_{0}$ and the temperature $T_{0}$ are dynamical variables depending only on the radius $r_{0}$ of the magnetic surface occupied, on the average by particle. For trapped particles, $n_{0}$ and $T_{0}$ are functions of the action variable $J_{3}$. In that case, it is easily proved that

$$
\frac{d F_{0}}{d J_{3}}=\frac{F_{0}(\mathbf{J})}{T_{0}}\left(\omega_{*}-\frac{d H_{0}}{d J_{3}}\right)
$$

where the diamagnetic frequency is given by

$$
\omega_{*}=T_{0}\left[\frac{1}{n_{0}} \frac{d n_{0}}{d J_{3}}+\frac{1}{T_{0}} \frac{d T_{0}}{d J_{3}}\left(\frac{H_{0}}{T_{0}}-\frac{3}{2}\right)\right] .
$$

We now introduce the Hamiltonian in the following form (with the assumption that $T_{0}$ is constant):

$$
H=H_{0}(J)+\bar{H}=\omega_{d}(\kappa ; s) \frac{E}{T_{0}} \psi+\hat{J}_{0} \tilde{U}=\omega_{d}(\kappa ; s) \frac{E}{T_{0}} \psi+\bar{U},
$$

where $\bar{U}=\hat{J}_{0} \widetilde{U}$ and $\hat{J}_{0}$ is the operator which transforms the fluctuating potential perturbation term $\widetilde{U}$ into its global gyroaverage (bounce motion plus cyclotron motions). The reduced gyrokinetic Vlasov equation is then given by

$$
\frac{d \bar{f}}{d t}=\frac{\partial \bar{f}}{\partial t}+\frac{d \phi_{3}}{d t} \frac{\partial \bar{f}}{\partial \phi_{3}}+\frac{d \psi}{d t} \frac{\partial \bar{f}}{\partial \psi}=0,
$$

where $\bar{f}=\bar{f}_{E, \kappa}\left(\psi, \phi_{3}, t\right)$ is the trapped ion distribution function. The characteristics are then given by the following equations:

$$
\begin{aligned}
& \frac{d \phi_{3}}{d t}=\omega_{d}(\kappa ; s) \frac{E}{T_{0}}+\frac{\partial}{\partial \psi}\left(\hat{J}_{0} \widetilde{U}\right), \\
& \frac{d \psi}{d t}=-\frac{\partial}{\partial \phi_{3}}\left(\hat{J}_{0} \widetilde{U}\right) .
\end{aligned}
$$

The self-consistency constraint is

$$
C_{e}\left(\frac{e \tilde{U}}{T_{0}}-\varepsilon_{e}\left\langle\frac{e \tilde{U}}{T_{0}}\right\rangle\right)-C_{i} \bar{\Delta}\left(\frac{e \tilde{U}}{T_{0}}\right)=\bar{n}_{i}-n_{p i},
$$

where $n_{p i}$ is the passing ion density and the gyroaveraged pseudodensity of trapped ions is given by

$$
\begin{aligned}
\bar{n}_{i}\left(\phi_{3}, \psi, t\right)= & \frac{2}{\sqrt{\pi}} \int_{0}^{\infty} d\left(\frac{E}{T_{0}}\right) \sqrt{\frac{E}{T_{0}}} \\
& \times \int_{0}^{\kappa_{\max }} d \kappa \kappa K(\kappa) \hat{J}_{0} \bar{f}_{\kappa, E}\left(\phi_{3}, \psi, t\right) .
\end{aligned}
$$

In previous expression we have $C_{e}=(1+\tau) / f_{p}$ and $C_{i}$ $=C_{e} f_{p} / \tau$, where $f_{p}=2 \sqrt{2 \varepsilon} / \pi$ is the fraction of trapped ions and $\tau=T_{i} / T_{e}$. The gyroaverage operator is $\hat{J}_{0}$ $=J_{0}\left(k_{\perp} \rho_{c}\right) J_{0}\left(k_{\psi} \delta_{b}\right)$ in the Fourier space, with $\rho_{c}$ and $\delta_{b}$ being the ion gyroradius and the ion banana width, respectively. We focus now on the features of the bounce and precession motions.

\section{B. The bounce motion}

For a trapped particle, as $d \phi_{2} / d t=\omega_{b}$, the revolution of the banana motion is completed within a time

$$
T_{b}=\frac{2 \pi}{\omega_{b}}=\int_{-\theta_{0}}^{+\theta_{0}} \frac{q_{0} R_{0} d \theta}{\sqrt{2 E / m_{i}} \sqrt{1-\lambda b(\theta)}},
$$

where $\quad 1-\lambda b(\theta)=m_{i} v_{\|}^{2} / 2 E, \quad \lambda=\mu B_{0} / E, \quad$ and $\quad E=\frac{1}{2} m_{i} v_{\|}^{2}$ $+\mu B_{0} b(\theta)$. Note that $v_{\|}=0$ leads to the angle limit $\theta_{0}$ given by $b\left(\theta_{0}\right)=1 / \lambda$. Using $b(\theta)=1+2 \varepsilon \sin ^{2}(\theta / 2)$ we have for the bounce frequency

$$
\omega_{b}=\sqrt{\frac{2 E}{m_{i}}} \frac{1}{q_{0} R_{0}} \bar{\omega}_{b}(\kappa ; s) .
$$

The previous integral can be easy determined by introducing the new variable $\kappa$ (the pitch-angle parameter) defined by

$$
\kappa^{2}=\sin ^{2}\left(\frac{\theta_{0}}{2}\right)=\frac{1-\lambda}{2 \varepsilon \lambda} .
$$

Trapped particles are characterized by $\kappa<1$, while $\kappa>1$ corresponds to passing ions. A little algebra gives the expression

$$
\bar{\omega}_{b}(\kappa) \simeq \frac{\pi \sqrt{\varepsilon}}{2 \sqrt{2}} \frac{1}{K(\kappa)},
$$

where $K(\kappa)$ and $E(\kappa)$ are the complete elliptic integral of the first and second kind, respectively.

\section{Toroidal precession motion}

The precession frequency $\omega_{3}=d \phi_{3} / d t=\omega_{d}$ is determined by the average over the bounce motion (i.e., over $\theta$ ). We have

$$
\omega_{d}=\frac{E}{e B_{0} R_{0}}\left[\left(\frac{d q}{d r}\right)_{r_{0}}\langle\theta \sin \theta\rangle_{\theta}+\frac{q\left(r_{0}\right)}{r_{0}}\langle\cos \theta\rangle_{\theta}\right] .
$$

We can write the frequency in the form 


$$
\omega_{d}(\kappa ; s)=\frac{q_{0} E}{r_{0} e B_{0} R_{0}} \bar{\omega}_{d}(\kappa ; s)=\omega_{d 0} \frac{E}{T_{0}} \bar{\omega}_{d}(\kappa ; s),
$$

where the dimensionless quantity is

$$
\bar{\omega}_{d}(\kappa ; s)=\frac{\int_{-\theta_{0}}^{+\theta_{0}} \frac{(s \theta \sin \theta+\cos \theta) d \theta}{\pi \sqrt{1-\lambda b(\theta)}}}{\int_{-\theta_{0}}^{+\theta_{0}} \frac{d \theta}{\pi \sqrt{1-\lambda b(\theta)}}} .
$$

Equation (33) can be simply derived by Kadomtsev in the form

$$
\bar{\omega}_{d}(\kappa ; s)=\frac{2 E(\kappa)}{K(\kappa)}-1+4 s\left(\frac{E(\kappa)}{K(\kappa)}+\kappa^{2}-1\right),
$$

where $s=\left(r_{0} / q_{0}\right)(d q / d r)_{r=r_{0}}$ is the magnetic shear. In a previous work, Fong and $\mathrm{Hahm}^{20}$ derived the nonlinear electrostatic bounce-averaged kinetic equations via phase space Lagrangian Lie-perturbation theory. They give the general form of the neoclassical polarization density term and take into account the contribution from the toroidal excursion in addition to the radial excursion. Our model uses a simplified expression of the neoclassical polarization density. However apart from the introduction of the drift frequency $\omega_{d} E$-term, Eq. (22) is a kinetic version of the Hasegawa-Mima (denoted here HM) model. ${ }^{21}$ Our model describes the resonant interaction between a wave and trapped ions via their precession motion. Equation (22) describes the ITG-driven turbulence and in particular the $\mathbf{E} \times \mathbf{B}$ nonlinearity. From these considerations, TIMs are expected to have an interchange modelike character with the possibility of inverse cascade. $^{22-24}$ Numerical simulations carried out here indicate that the polarization drift term do not play a major role in the initial phase of growth of the streamer-type turbulence. However a previous work ${ }^{25}$ indicated that the polarization nonlinearity may modify the transport scaling. The effect of the polarization term will be investigated in Sec. VI B.

\section{CONNECTION WITH THE HASEGAWA-MIMA MODEL}

It is well known that theoretical understanding of zonal flows and streamers in the context of tokamak plasmas has started with studies based on the drift-wave model and, in particular, the HM model. In toroidal geometry, it must be pointed out that the simplest model that allows the study of instabilities induced by electrostatic drift waves is that of Hasegawa and Watakani. ${ }^{26}$ In its classic form, the HM equations $^{21}$ read in the slab $(x, y)$ geometry,

$$
\begin{aligned}
& \frac{d}{d t}\left(U-\nabla_{\perp}^{2} U-x\right)=0, \\
& \frac{d}{d t}=\frac{\partial}{\partial t}-\frac{\partial U}{\partial y} \frac{\partial}{\partial x}+\frac{\partial U}{\partial x} \frac{\partial}{\partial y} .
\end{aligned}
$$

Here the time $t$, the space variables $x$ and $y$, and the electrostatic potential $U=U(x, y, t)$ are normalized appropriately. Usually streamers (modes with $k_{x}=0$ in the slab geometry) can be generated even by the linear instability mechanism (although the nonlinear excitation is also possible), whereas a zonal flow structure (the mode with $k_{y}=0$ ) can be generated only through nonlinear processes. Large-scale structures (including such zonal flows and/or streamers) are nonlinearly generated through the three-wave coupling modulation in turbulent fluctuations. The mechanism is based on the triad interactions and is attributed to the development of a modulation, or parametriclike type instability induced by the small-scale turbulence.

It is worth pointing out, at this stage, that the HM model described above contains two nonlinear terms. The first one is the $\mathbf{E} \times \mathbf{B}$ convective nonlinearity leading to a forward energy cascade into the shorter scale lengths. The polarization drift nonlinearity, appearing by the term $\Delta_{\perp} U$, is well studied in gyrokinetic modeling and is known to cascade power toward long scales (indeed an inverse enstrophy cascade). Thus the nonlinear generation of large-scale streamertype structures (here denoted as LSSS), at the saturation process, may be governed by a symmetry breaking mechanism which will ensure selective condensation of power into one of these two shear flow. Such a tendency to generate largescale modes has been already observed in plasma turbulence. $^{27}$ For instance, in flute turbulence, ${ }^{28}$ such largescale structures are called the generalized Kelvin-Helmholtz modes.

Several remarks must be pointed out.

A more realistic description of plasma turbulence ${ }^{29}$ on ion-gyroradius scales necessitates the introduction of a mean potential $\langle U\rangle_{y}$ leading to

$\frac{d}{d t}\left(U-\langle U\rangle_{y}-\nabla_{\perp}^{2} U-x\right)=0$.

Indeed Eq. (37), in its generalized form, allows the description of systems with generation of strong zonal flows $\left(k_{y}=0\right)$ in the ITG case, while the classic HM equation (35) describes a system in which streamers (with $k_{x}=0$ ) can be sustained in ETG. Indeed the last case is also probably relevant to TEM turbulence. Although one of the stabilizing effects of TIM turbulence is the negative magnetic shear, there are only few dedicated investigations concerning the streamer regime of the TIM turbulence.

(ii) It is well known that ITG or ETG instabilities may grow exponentially until a nonlinear saturation mechanism sets in. Such one saturation process is the onset of so-called secondary instabilities. In particular, the role of these secondary instabilities in the formation of zonal flows or streamers is shown in Ref. 30. It was proposed recently ${ }^{31}$ that there is a relation between such secondary instabilities with a modulational instability involving a four-mode coupling. Such a modulational instability involves a pump wave (describing here an initial streamer) $U_{q}$ with $\mathbf{k}_{q}$ given by $\left(k_{x}, k_{y}\right)=(0, q)$, a zonal flow $U_{0}$ with wave vector $\mathbf{k}_{0}=(p, 0)$, and two sidebands $U_{ \pm}$with wavevectors $\mathbf{k}_{ \pm}=(p, \pm q)$. According to the classic wavevector and frequency matching conditions, these modes are allowed to nonlinearly interact with each other in dif- 
ferent ways. In particular, the zonal flow $\left(k_{y}=0\right)$ or the streamer $\left(k_{x}=0\right)$ mode is only a special component of general secondary fluctuations large-scale structures.

(iii) A crucial issue is to understand whether the zonal flow or streamer in ITG or ETG turbulence is preferentially generated. Recently, a physical mechanism was identified ${ }^{31}$ as the governing role of the magnetic shear: The nonlinear formation of large-scale structures such as zonal flow, streamers, or LSSS modes can be controlled by the magnetic shear. The existence of different regimes of interaction seems to indicate the possibility of a bifurcationlike mechanism depending on the magnetic shear parameter.

(iv) The model equation describing the ion dynamics in ITG mode is essentially a HM equation while TIMs are described here in Eqs. (22)-(25) using a reduced kinetic Vlasov description. Consequently, the dissipation mechanism, which is a key element in the turbulent flow, is different in our model. Note that the presence of the polarization drift nonlinearity is a key element for the growth of the instability in the HM model. In the reduced Vlasov model describing the TIM turbulence, this term is not required to start up the instability. Note that the streamers also suffer from the Landau damping so that zonal flow may become more unstable in a realistic plasma. In particular, using a drift-kinetic Vlasov model, Jenko and Scott ${ }^{32}$ showed that the Landau damping is associated with parallel trapping to reduce the transport for low magnetic shear $(s<1)$, whereas it plays a very weak role for higher shear $(s>1)$.

\section{LINEAR ANALYSIS OF THE TIM INSTABILITY}

In order to provide a simple physical picture of the TIM turbulence in the linear regime, the dispersion relation was obtained by linearizing Eqs. (22) and (25), using relations (23) and (24) for the time derivative with (we have omitted the "barr" notation here to simplify the presentation)

$$
\begin{aligned}
& f_{\kappa, E}\left(\phi_{3}, \psi, t\right)=F_{0}(\psi)+\sum_{n} \tilde{f}_{n}(\psi) e^{i\left(n \phi_{3}-\omega t\right)}, \\
& \tilde{U}=U_{0}(\psi)+\sum_{n} \widetilde{U}_{n}(\psi) e^{i\left(n \phi_{3}-\omega t\right)},
\end{aligned}
$$

where the equilibrium distribution function and potential are taken as (the energy $E$ being normalized to $T_{0}$ )

$$
\begin{aligned}
& F_{0}(\psi)=e^{-E}\left[1+\Delta \tau \omega_{d}(\kappa ; s)\left(E-\frac{3}{2}\right) \psi\right], \\
& U_{0}(\psi)=0 .
\end{aligned}
$$

Here $\Delta \tau$ is the normalized temperature gradient $\left(\Delta \psi / T_{0}\right)$ $\times\left(d T_{0} / d \psi\right)$. Two remarks must be pointed out.

(i) First it is possible to determine the marginal solution for the electric potential fluctuations. The reduced Vlasov equation (22), together with the quasineutrality condition equation (25), gives the condition (assuming the polarization term negligible in first approximation)

$$
C_{e} \widetilde{U}_{n}=\frac{2}{\sqrt{\pi}} \int_{0}^{\infty} d E \sqrt{E} \int_{0}^{1} d \kappa \kappa K(\kappa) \frac{n \Delta \tau e^{-E}\left(E-\frac{3}{2}\right)}{E-\frac{\omega}{n \omega_{d}(\kappa ; s)}} \widehat{J}_{0} \widetilde{U}_{n},
$$

with the usual Landau prescription on the imaginary part of $\omega$. In the linear regime the imaginary part of Eq. (42) must cancel exactly for the marginal solution, which implies that the dispersion relation for TIM is given by

$\omega=\frac{3}{2} n\left\langle\omega_{d}\right\rangle_{\kappa}$.

Equation (42) reduces then to:

$\tilde{U}_{n}-\frac{\Delta \tau}{C_{e}} \frac{2}{\sqrt{\pi}} \int_{0}^{\infty} d E \sqrt{E} \int_{0}^{1} d \kappa \kappa K(\kappa) e^{-E} \hat{J}_{0}^{2} \widetilde{U}_{n}=0$,

where $\hat{J}_{0}$ is approximated to

$\hat{J}_{0}=\left[1+\frac{1}{4} E \delta_{b}^{2} \partial_{\psi}^{2}\right]\left[1+\frac{1}{4} E \rho_{c}^{2} \partial_{\phi_{3}}^{2}\right]$.

The ITG-like instability presents a threshold for trapped ions given by

$\Delta \tau_{s}=\frac{C_{e}}{\xi}$,

where

$\xi=\frac{2}{\sqrt{\pi}} \int_{0}^{\infty} d E \sqrt{E} \int_{0}^{1} d \kappa \kappa K(\kappa) e^{-E} J_{0}^{2}\left(\pi \delta_{b} \sqrt{E}\right) J_{0}^{2}\left(\pi \rho_{c} \sqrt{E}\right)$.

It is then possible to obtain the marginal solution by solving Eq. (44). For zero boundary conditions, we obtain

$\tilde{U}_{n}(\psi)=\sin (l \pi \psi) \quad$ with $\quad l=1,2,3, \ldots$.

The knowledge of this marginal solution allows us to start the ITG-type instability at a very low level. For instance, for $l=1$ and $\rho_{c}=0$ the ITG instability threshold is given by

$\Delta \tau_{s}=\frac{C_{e}}{1-\frac{3}{4}\left\langle\delta_{b}^{2}\right\rangle_{\kappa}+\frac{15}{64}\left\langle\delta_{b}^{4}\right\rangle_{\kappa}}$.

For instance, for typical fusion parameters using $C_{e}$ $=0.10$ the threshold is then $\Delta \tau_{s} \simeq 0.1000740$ very close to the normalized value of $C_{e}$.

(ii) It is possible to obtain the (kinetic) dispersion relation using the standard Landau analysis. For instance, for $l=1$, which corresponds to the lowest gradient, the dispersion relation can be expressed as follows [with a frequency shift given by $\Delta \omega=\omega / n-\frac{3}{2} \omega_{d}(\kappa ; s)$ and assuming that $\hat{J}_{0}^{2} \simeq 1$ ]: 

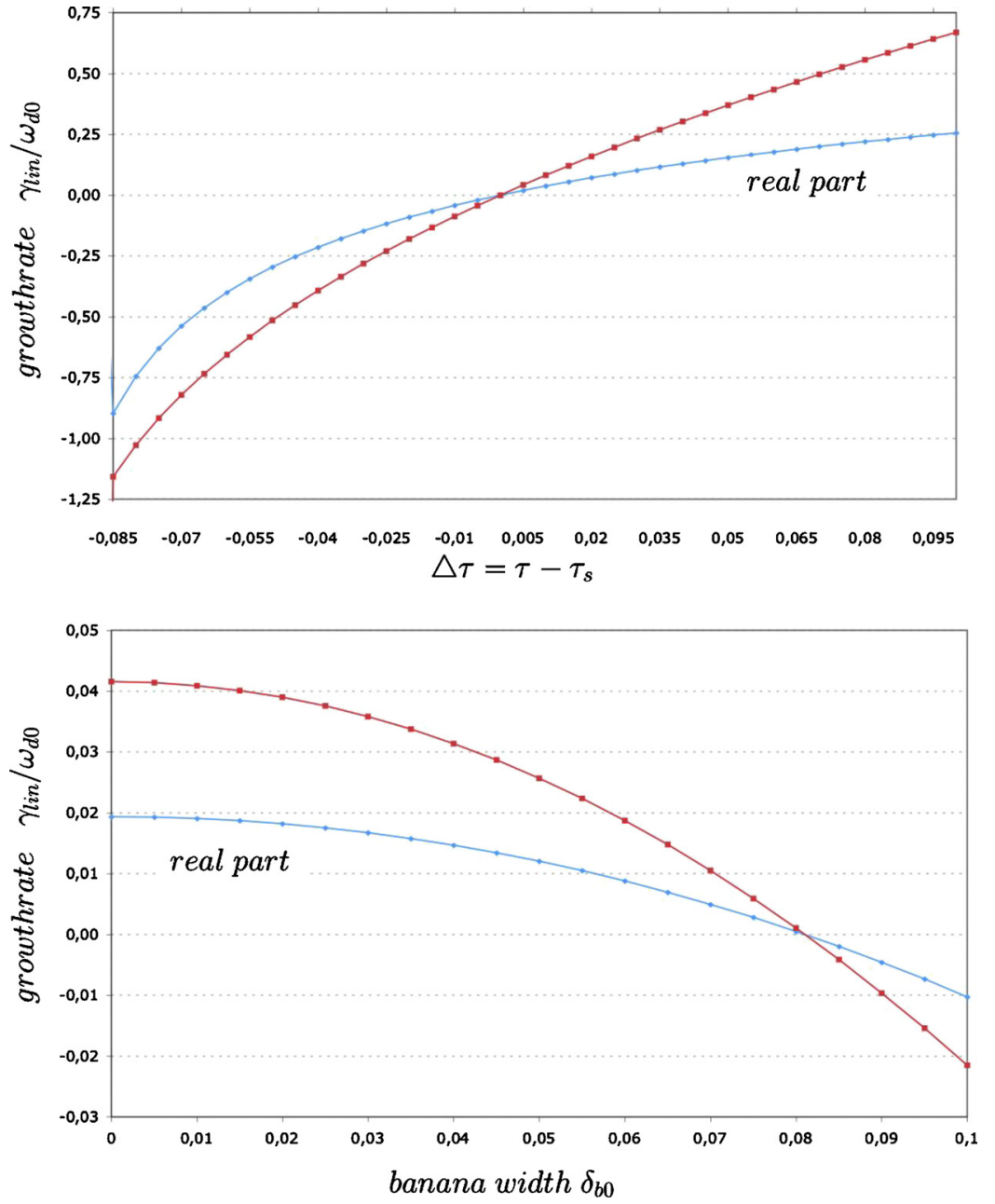

FIG. 1. (Color online) Linear growthrate and real frequency of the driven ITG instability for trapped ion modes as a function of the quantity $\Delta \tau-\Delta \tau_{s}$ (on the top panel) and as a function of the "banana width" $\delta_{b}$ (on the bottom panel).

$$
\begin{aligned}
\frac{1}{2}\left(\frac{\Delta \tau_{s}}{\Delta \tau}-1\right)= & \int_{0}^{1} d \kappa \frac{\kappa K(\kappa)}{\omega_{d}(\kappa ; s)}\left[1+\sqrt{\frac{3}{2}+\frac{\Delta \omega}{\omega_{d}(\kappa ; s)}}\right. \\
& \left.\times Z\left(\sqrt{\frac{3}{2}+\frac{\Delta \omega}{\omega_{d}(\kappa ; s)}}\right)\right],
\end{aligned}
$$

where $Z$ is the Fried and Conte function. This relation has been solved numerically with $C_{e}=0.1, \delta_{b} / \Delta \psi$ $=0.01$. The result is shown in Fig. 1. The top panel in Fig. 1 shows the growthrate $\gamma_{\mathrm{th}} / \omega_{d 0}$ and the real frequency $\omega / \omega_{d 0}$ as a function of the parameter $\Delta \tau-\Delta \tau_{s}$. We see clearly that the instability occurs only above a given threshold. The bottom panel in Fig. 1 represents the evolution of the growthrate $\gamma_{\mathrm{th}} / \omega_{d 0}$ as a function of the banana width $\delta_{b}$ (assumed here to be fixed) for a given value of $\Delta \tau-\Delta \tau_{s}=0.0025$ using a threshold of $\Delta \tau_{s} \simeq 0.1000740$. We see the possibility of stabilization of the instability when $\delta_{b}$ reaches the value of $\delta_{b l}=0.08 \Delta \psi$.

Next, we would like to discuss what happens if we take into account the $\kappa$-dependence in the drift precession frequency $\omega_{d}$. This $\kappa$-dependence in $\omega_{d}$ is given by relation (34) which defines the dimensionless quantity $\bar{\omega}_{d}(\kappa ; s)$. The $\kappa$-dependence allows the introduction of the magnetic shear parameter $s$. The magnetic shear describes the dependence of field line direction on the coordinate across closed flux surfaces. Its magnitude varies both along and transverse to the field lines. The longitudinal variation traps particles and leads to the resulting physical picture of a banana orbit of the projection of the guiding center orbit onto the cross section at fixed toroidal angle. The transverse variation, referred usually as the magnetic shear, has stabilization effects on collective instabilities. Figure 2, on the top panel, shows the behavior of the quantity $\bar{\omega}_{d}(\kappa ; s)$ as a function of the pitchangle parameter $\kappa$, for different values of the magnetic shear $s(-2 \leq s \leq 2)$. We observe the possibility to obtain negative values of $\bar{\omega}_{d}(\kappa ; s)$ when the magnetic shear is introduced. According to Kadomtsev and Pogutse, ${ }^{12}$ the stabilization of trapped particle driven instabilities takes place through the reversal of the precession drift. In the bottom panel in Fig. 2, we have plotted the behavior of the growthrate of the TIM instability as a function of $s$ in the linear regime: We see clearly that $\gamma_{\mathrm{th}} / \omega_{d 0}$ decreases for small values of the magnetic shear and the beginning of a stabilization process when $s \leq 0.4$.

Such a result confirms the possibility of stabilization proposed recently ${ }^{33}$ where the authors proposed that the collisionless TEM instability may be suppressed in the negativeshear region due to the reversal of the toroidal precessional drift of trapped electrons. 

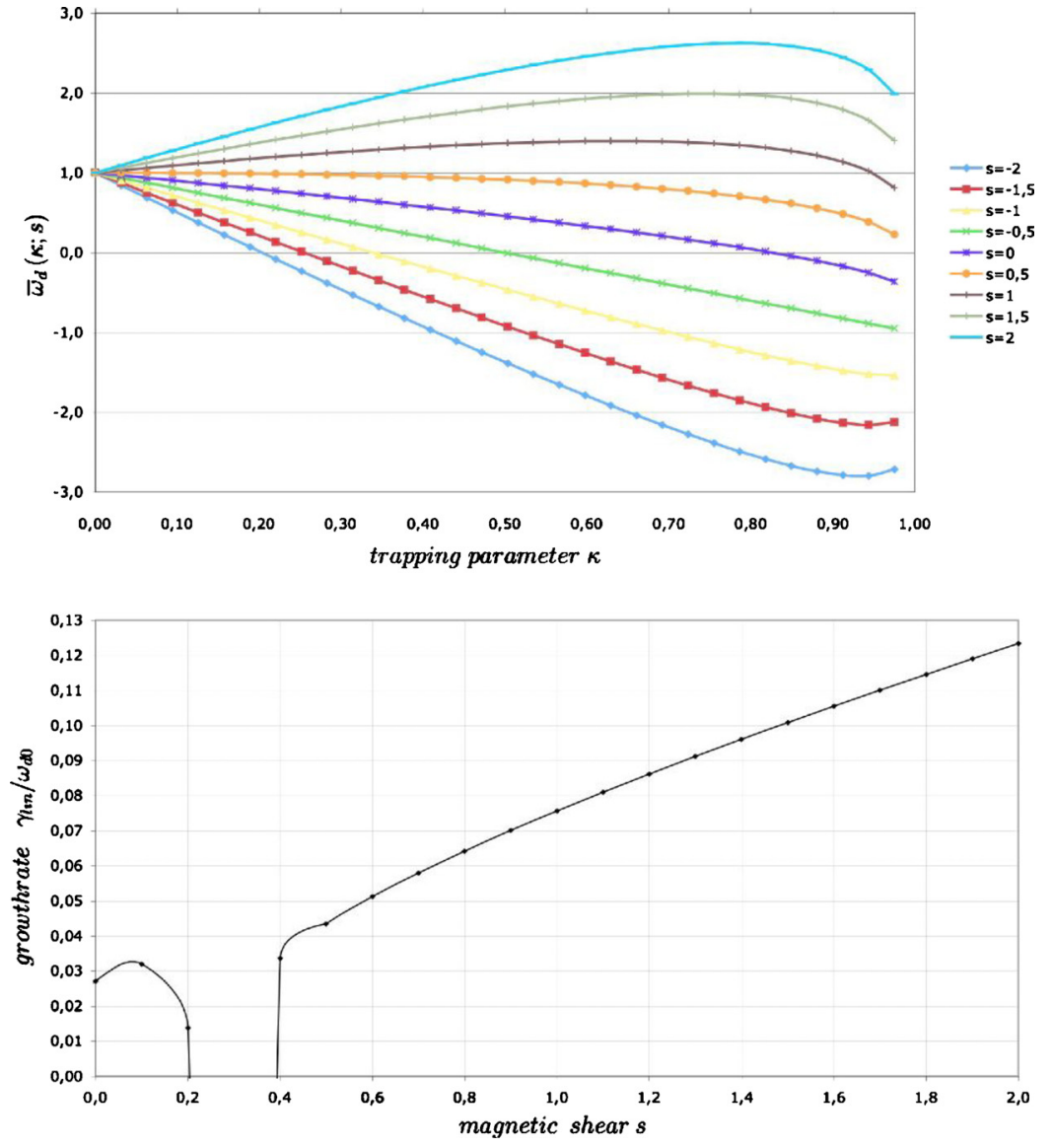

FIG. 2. (Color online) On the top panel a set of curves representing the dimensionless drift frequency $\bar{\omega}_{d}$ as a function of the trapping parameter $\kappa$ for different values of the magnetic shear. On the bottom panel, the behavior of the linear growthrate as a function of the magnetic shear $s$.

\section{THE NUMERICAL SCHEME}

The numerical resolution of the Vlasov kinetic equations is generally performed using particle-in-cell methods, which provide satisfying results with relatively few particles. However, for some applications, in particular, when resonant particles play an important role or when the numerical noise due to the finite number of superparticles becomes too important, another method must be found. A complementary approach is to adopt an Eulerian point of view and to represent $f$ on a grid in two-dimension space in $\left(\psi, \phi_{3}\right)$.

Another way, which we have adopted here, is by combining the Eulerian and the Lagrangian approaches into a "semi-Lagrangian" (SL) scheme which traces back the trajectories at every time step, always starting from the regular "particle" distribution in the $\left(\psi, \phi_{3}\right)$ space. Thus the idea behind semi-Lagrangian advection is to try to get the best of both approaches: the regular resolution of Eulerian schemes and the enhanced stability of the Lagrangian ones. This is achieved by using a different set of particles being chosen at the beginning of the time step such that they arrive exactly at the mesh points of the grid at the end of the time step. The method is usually based on the well-known time splitting scheme first introduced by Knorr and Cheng. ${ }^{34}$ Before considering the case of the TIM turbulence, we adopt to outline here some of the physical meaning of this method in the case of the study of an electrostatic one-dimensional plasma. We want to solve the (normalized) Vlasov equation for the (electron here) distribution function $f(x, v, t)$,

$$
\frac{\partial f}{\partial t}+v \frac{\partial f}{\partial x}+E(x, t) \frac{\partial f}{\partial v}=0
$$

where the electric field $E(x, t)$ is given self-consistently by the Poisson equation

$$
\frac{\partial E}{\partial x}=\int_{-\infty}^{+\infty} f(x, v, t) d v-1
$$

starting from a given initial condition $f(x, v, t=0)$. Note that in the electrostatic case considered here, $x, v$, and $t$ are normalized to the Debye length, the thermal velocity, and the inverse plasma frequency $\omega_{p}^{-1}$, respectively. The Vlasov equation (51) is then integrated in the original phase space by applying a splitting scheme (indeed a fractional step method), which consists of treating the convective term $v \partial f / \partial x$ and the acceleration term $E \partial f / \partial v$ separately. The crucial point is the representation of the electric field $E(x, t)$ by a succession of Dirac pulses $E^{*}(x, t)=E(x, t) \Delta t \Sigma_{n} \delta(t$ $\left.-t_{n+1 / 2}\right)$, where $t_{n+1 / 2}=(n+1 / 2) \Delta t$. Thus the numerical integration of the Vlasov-Poisson system is obtained by the following sequence of shifts:

$$
\begin{aligned}
& f^{*}(x, v)=f^{n}\left(x-\frac{v \Delta t}{2}, v\right), \\
& f^{* *}(x, v)=f^{n}\left[x, v-E\left(x, t_{n+1 / 2}\right) \Delta t\right],
\end{aligned}
$$




$$
f^{n+1}(x, v)=f^{* *}\left(x-\frac{v \Delta t}{2}, v\right),
$$

where the electric field in Eq. (54) is obtained by solving the Poisson equation (52) just after step (53). It must be pointed out that substituting successively Eqs. (53) and (54) into Eq. (55) leads to

$$
f^{n+1}(x, v)=f^{*}\left[x-\Delta t\left(v-\frac{1}{2} E(\widetilde{x}) \Delta t\right), v-E(\widetilde{x}) \Delta t\right],
$$

with $\tilde{x}=x-v \Delta t / 2$. Thus we find that Eq. (56) is equivalent to the following integrated equations of the characteristics of the Vlasov equation (51) by comparing the arguments of $f^{*}$ and we have

$$
\begin{aligned}
& x_{n}=x_{n+1}-\Delta t\left[v_{n+1}-E\left(x_{n+(1 / 2)}, t_{n+(1 / 2)}\right) \frac{\Delta t}{2}\right], \\
& v_{n}=v_{n+1}-\Delta t E\left(x_{n+(1 / 2)}, t_{n+(1 / 2)}\right) .
\end{aligned}
$$

The continuum form of the characteristic equations is of course given by

$$
\frac{d x}{d t}=v \quad \text { and } \quad \frac{d v}{d t}=E[x(t), t] .
$$

Thus, from a numerical point of view, the splitting scheme is equivalent to an integration of the Vlasov equation along their characteristics and is correct to the second order in $\Delta t$. However, from a physical point of view, it is very important to understand how the SL model incorporates some basic plasma properties such as the linear theory, in particular, for a future study of the turbulence in plasmas. For instance, linearizing Eq. (51) with $E^{*}$ around a homogeneous equilibrium [i.e., $\left.f(x, v, t)=n_{0} F_{0}(v)+f_{1}(x, v, t)\right]$ and performing the usual Laplace $(t \rightarrow s)$ and Fourier $(x \rightarrow k)$ transforms yields ${ }^{35}$ to

$$
\begin{aligned}
-i k\left[E(k, s)+\frac{i}{k} \sum_{n} E\left(k, s-\frac{2 i \pi n}{\Delta t}\right) \int_{-\infty}^{+\infty} \frac{d F_{0}}{d v} \frac{d v}{s-i k v}\right] \\
=\int_{-\infty}^{+\infty} \frac{f_{1}(k, v, 0) d v}{s-i k v} .
\end{aligned}
$$

However, the appearance of the sum $\Sigma_{n}$ on the left-hand side of Eq. (60) does not allow us to recover directly the plasma dispersion relation. However since we know that the frequency spectrum of the electric field does not exceed a few $\omega_{p}$ [choosing $\Delta t$ such that $\Delta t \omega_{p} \ll 1$ results in a zero contribution of all terms of the sum on the left-hand side of Eq. (60), except for $n=0$ which recovers the usual Landau dispersion relation].

This method can be then applied to the treatment of the gyrokinetic Vlasov equation (22). Here Poisson equation (52) is replaced by the quasineutrality condition (25). Rewriting the gyropotential $\hat{J}_{0} \widetilde{U}$ in the form of a Dirac pulse $\hat{J}_{0} \widetilde{U}^{*}=\Sigma_{n} \Delta t J_{0} \tilde{U} \delta\left(t-t_{n+1 / 2}\right)$, the numerical integration scheme is then given by the following sequence:

$$
f^{*}\left(\psi, \phi_{3}\right)=f^{n}\left(\psi, \phi_{3}-\frac{\omega_{d}(\kappa ; s) E \Delta t}{2}\right),
$$

$$
f^{* *}\left(\psi, \phi_{3}\right)=f^{*}\left(\psi+\frac{\partial}{\partial \phi_{3}}\left(\hat{J}_{0} \tilde{U}^{+}\right) \Delta t, \phi_{3}-\frac{\partial}{\partial \psi}\left(\hat{J}_{0} \tilde{U}^{+}\right) \Delta t\right),
$$

$$
f^{*}\left(\psi, \phi_{3}\right)=f^{n}\left(\psi, \phi_{3}-\frac{\omega_{d}(\kappa ; s) E \Delta t}{2}\right),
$$

where $\hat{J}_{0} \tilde{U}^{+}=\hat{J}_{0} \tilde{U}\left[\phi_{3}-\left(\left[\omega_{d}(\kappa ; s) E \Delta t\right] / 2\right), \psi, t_{n+1 / 2}\right]$ and the second step is integrated along the characteristics without time splitting, $f^{* *}$ is then interpolated using a tensor product of cubic B-splines (i.e., a direct two-dimensional advection scheme). This corresponds to use

$$
\begin{aligned}
& \psi_{n}=\psi_{n+1}+\frac{\partial}{\partial \phi_{3}}\left(\hat{J}_{0} \tilde{U}^{+}\right) \Delta t, \\
& \phi_{3, n}=\phi_{3, n+1}-\omega_{d}(\kappa ; s) E \Delta t-\frac{\partial}{\partial \psi}\left(\hat{J}_{0} \tilde{U}^{+}\right) \Delta t,
\end{aligned}
$$

for the numerical integration of the Vlasov equation (22) along the characteristics given by Eqs. (24) and (23).

We have also introduced a transition region where the potential fluctuations are damped at box boundaries. A buffer region then is introduced at the boundaries in $\psi$. These fluctuations at the boundaries are controlled by a diffusion coefficient $D$. Thus in practice, the right-hand side of the Vlasov equation (22) is replaced by the term $\partial_{\psi}\left(D \partial_{\psi} \bar{f}\right)$, where the diffusion coefficient $D(\psi)$ is zero except in the buffer region.

Some remarks are worth noting:

(i) To keep the accuracy of the second order in time, time splitting between the phase space coordinates $\left(\psi, \phi_{3}\right)$ in the second equation (62) of the sequence of integration is not allowed in our Vlasov equation ${ }^{36}$ because the advection field in each resulting split equation would be not divergence free. Therefore a direct two-dimensional advection is required. It is not the case for the global sequence equations (61)-(63).

There are two main reasons for considering this SL approach instead of particle methods. First the Vlasov method does not produce numerical noise, an advantage that is particularly important with respect to trapped particle dynamics in turbulent regime since we can theoretically start up the ITG instability from the round-off errors of the computer. This allows us to study primary parametriclike three-wave interaction scenario as a possible path toward the fully developed turbulence. Second, it is made sure that all relevant regions of phase space are well represented. For example, the low-density phase space distribution function keeps the same sampling allowing a good description of the Landau resonance or wave-trapped particles resonance in the precession motion of particles. Even if the use of action-angle variable leads to a more accurate description of wave-particle interaction in a numerical point of view, the accuracy of the SL model allows us to follow the interaction of a weak number of modes without the excitation of all the spectrum when the numerical noise is too high. 
(iii) In gyrokinetic simulations (in particular, when involving five-dimensional phase space), attempts to further reduce the numerical demands have proven to be challenging for ITG or TIM turbulence. Efficiency may be improved by the use of $(\mathbf{J}, \boldsymbol{\phi})$ action-angle variables which remove the constraint of explicit Vlasov solvers to resolve the fastest dynamics in the plasma even if it does not affect the accuracy. Here two independent variables $\left(\phi_{1}, \phi_{2}\right)$ corresponding to the gyrophases have been removed and two of the remaining variables $E, \kappa$ (or more generally $E$ and $\mu$ ) enter the equations only as parameters, which reduce the associated numerical effort. This makes possible the simulation on long time scales. The second advantage is a direct parallelization along the invariants $E$ and $\kappa$. However numerical Vlasov simulations have shown that it is necessary to keep a sufficient number of invariants in order to keep a high accuracy and to control the start up of the instability. Indeed the more the phase space sampling or the number of invariants is high, the best is the control of the instability.

(iv) The last point concerns the competition process between the growth of the linear modes described by the linear theory of the ITG instability and the toroidal nonlinear coupling obtained in the framework of the parametric three-oscillator model or the modulational instability. Provided that the initial broadening of the frequency spectrum is not too large, the model allows a very description of the standard Landau dispersion relation. However in the developed turbulence regime, Vlasov simulations have shown that more than about 100 invariants in energy are required to follow accurately the initial phase of the instability and to separate the linear aspect of the instability growth and the nonlinear coupling which arises near the threshold of the instability.

\section{TIM TURBULENCE BEHAVIOR IN PARTICULAR REGIMES OF DYNAMICAL INTEREST}

\section{A. Nonlinear Vlasov simulation at moderate magnetic shear $(s=0.5)$}

The starting point for a systematic investigation of TIM turbulence is the gyrokinetic Vlasov model coupled with the quasineutrality condition initiated in an equilibrium state with a perturbed term. Numerical simulations were carried out using our SL Vlasov solver, in the case of an initial distribution function given by

$f_{\kappa, E}\left(\phi_{3}, \psi, t=0\right)=e^{-E}\left[1+\Delta \tau\left(E-\frac{3}{2}\right) \psi \omega_{d}(\kappa ; s)+\Delta \tau \hat{J}_{0} U\right]$,

where $\widetilde{U}=U_{0} \sin (\pi \psi) \cos \left(10 \phi_{3}\right)$ is the initial electrostatic potential perturbation. In simulation normalized quantities were used: The time is normalized to the inverse drift frequency $\omega_{d 0}^{-1}$, the poloidal flux is given in $\Delta \psi$ units (with $\Delta \psi$ $\left.=r_{0} R_{0} B_{0} / q_{0}\right)$, the energy $E$ is given in $T_{0}$ unit, and the potential is expressed in $T_{0} / e$ unit (note we have indeed the condition $\left.\omega_{d 0} \Delta \psi=T_{0} / e\right)$.
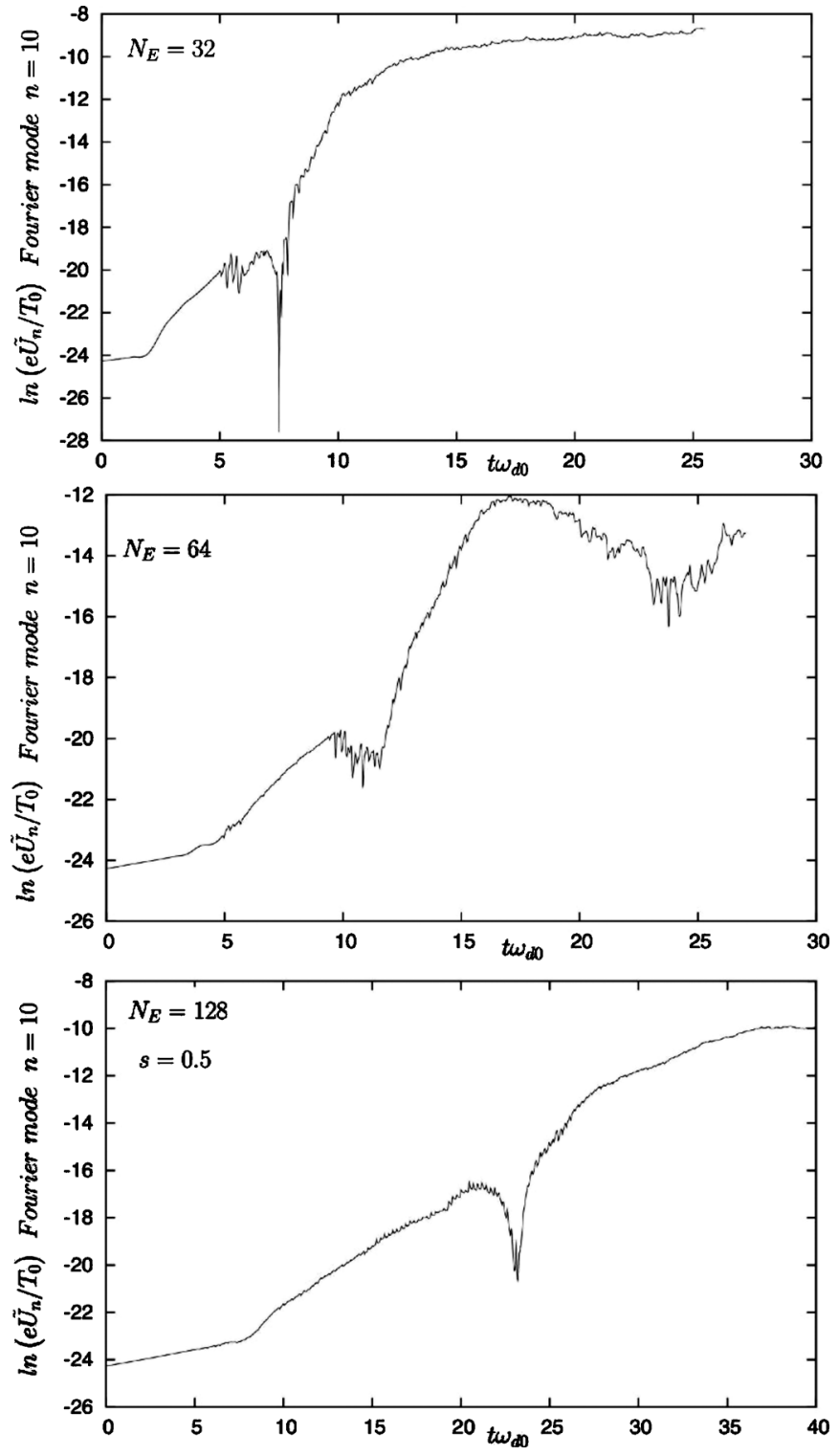

FIG. 3. Time evolution of the Fourier mode $n=10$ of the electric potential, in a logarithmic scale for different values of the number of invariants in energy. This mode is initially excited at a weak level and grows then in time. The numerical simulation performed with a positive and moderate magnetic shear $(s=0.5)$.

For now, we would like to restrict to quasineutrality condition without polarization term, various generalizations will follow later in Sec. VI B. Simulations were carried out with a temperature gradient $\Delta \tau$ just above the threshold of the ion trapped instability. We have used here $\Delta \tau-\Delta \tau_{s}=0.00750$ with a value of the threshold of $\Delta \tau_{s}=0.1000740$. We take a banana width of $\delta_{b} / \Delta \psi=0.010$ and a magnetic shear of $s$ $=0.50$. The phase space sampling is then $N_{\psi}=128$ and $N_{\phi_{3}}$ $=512$. We choose to keep fixed the sampling in $\kappa$ (pitchangle parameter) $\left(N_{\kappa}=16\right)$ and perform three simulations for different sampling in energy: $N_{E}=32,64$, and 128 values of the "adiabatic" invariant $E$.

Figure 3 shows the temporal evolution of the initially perturbed mode $\left\langle e \widetilde{U}_{n=10} / T_{0}\right\rangle_{\psi}$, averaged over the poloidal flux $\psi$, in a logarithmic scale, for three different values of the 

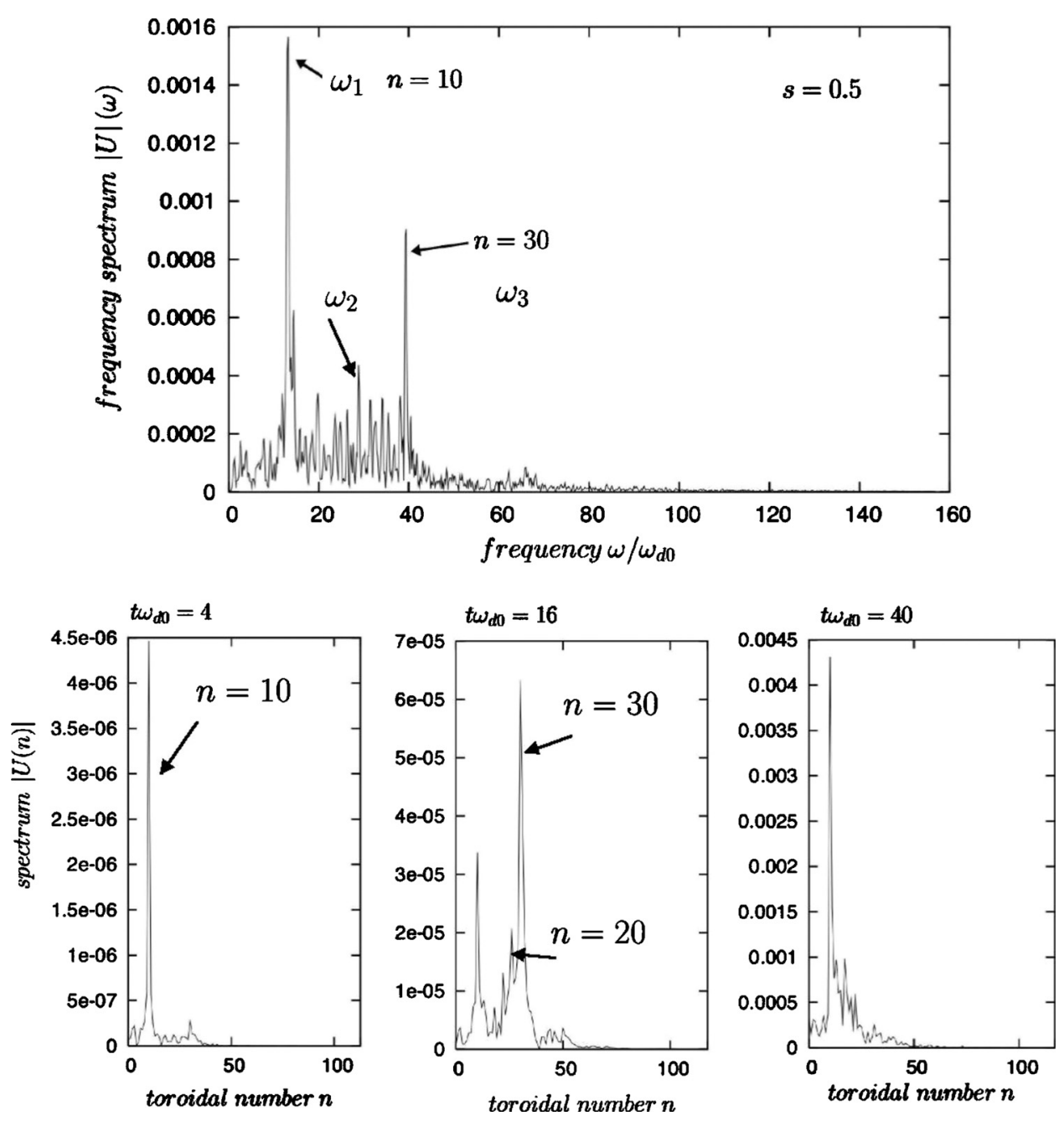

FIG. 4. Continuing the presentation of results of simulation shown in Fig. 3, the frequency spectrum (on the top panel) exhibits two dominant modes, verifying the standard dispersion relation $\omega=3 n\left\langle\omega_{d}\right\rangle / 2$. The corresponding spectrum in toroidal number of the potential is shown at bottom panels for three different times at each step of the evolution. Note the damping of the second dominant mode at the saturation at time $t \omega_{d 0}=40$. energy invariant. The evolution exhibits linear properties: The mode $n=10$ matches the respective linear phase at the beginning of the growth. The curves exhibit three different phases or regimes in time. If one starts out with a density perturbation amplitude well below the unity (close to $10^{-4}$ ), the system first goes through a linear phase in which the nonlinear terms are negligible during this step and the various modes in the system remain uncoupled to each other. We observe that it is possible to follow the linear phase for a much longer time by increasing the number of invariants in energy. The top panel corresponds to $N_{E}=32$, the middle panel to $N_{E}=64$, while the bottom panel is obtained for $N_{E}$ $=128$. The linear numerical growthrate is close to $\gamma_{\text {num }} / \omega_{d 0}$ $\simeq 0.050$ in fair agreement with the theoretical value of $\gamma_{\mathrm{th}} / \omega_{d 0} \simeq 0.045$ obtained by solving the dispersion relation (50). As expected, it turns out that the system is quickly dominated by the excited mode $n=10$, initially perturbed here. Although the other modes are also excited, they remain at a very weak level due to the very low level of numerical noise afforded by the SL model. The energy is tapped via the linear ITG instability and set into the other modes: The interaction leads to the growth of the mode $n=30$ (close to the third harmonic), which rapidly becomes the dominant mode (not shown here). This mechanism of nonlinear mode coupling modifies the structure of the mode $n=10$ in time and leads to the increase of the growthrate. This nonlinear mode coupling forms the second phase of the instability, followed by the saturation regime.

The spectrum in frequency is shown on the top panel in Fig. 4 for $N_{E}=128$. We see clearly that the two modes are dominant here: The first one corresponds to the initial perturbation (the driving term) at a frequency close to $\omega_{1}$ $=\frac{3}{2} n_{1}\left\langle\omega_{d}\right\rangle_{\kappa} \simeq 13 \omega_{d 0}$ choosing the mode $n_{1}=10$ and a value of $\left\langle\omega_{d}\right\rangle_{\kappa} \simeq 0.866$. The second peak corresponds to the mode $n_{3}$ $=30$ and is close to $\omega_{3} \simeq 39.5 \omega_{d 0}$. The excitation of the mode $n_{3}=30$ is clearly visible at the bottom panel in Fig. 4, which has been plotted at time $t \omega_{d 0}=16$, well in the region of the nonlinear coupling. The corresponding frequency seems in good agreement with the expected value of $\omega_{3}=\frac{3}{2} n_{3}\left\langle\omega_{d}\right\rangle_{\kappa}$ $\simeq 39 \omega_{d 0}$ using $\left\langle\omega_{d}\right\rangle_{\kappa} \simeq 0.866$. We observe also the presence of a weaker mode $\omega_{2} \simeq 27 \omega_{d 0}$, which seems to indicate a three-wave parametriclike coupling with the mode $n_{2}=20$ (i.e., having a linear frequency of $\omega_{2}=\frac{3}{2} n_{2}\left\langle\omega_{d}\right\rangle_{\kappa} \simeq 26 \omega_{d 0}$ in well agreement with the observed value in the spectrum). The bottom panels in Fig. 4 corresponds to the plot of the spectrum of the potential fluctuations $\left\langle e \tilde{U} / T_{0}\right\rangle_{\psi}$ as a function of the toroidal number. At left, plotted at time $t \omega_{d 0}=4$, we observe the initial perturbation mode growth on $n_{1}=10$, the other modes being negligible. At time $t \omega_{d 0}=16$ (middle bot- 
$t \omega_{d 0}=2.5$
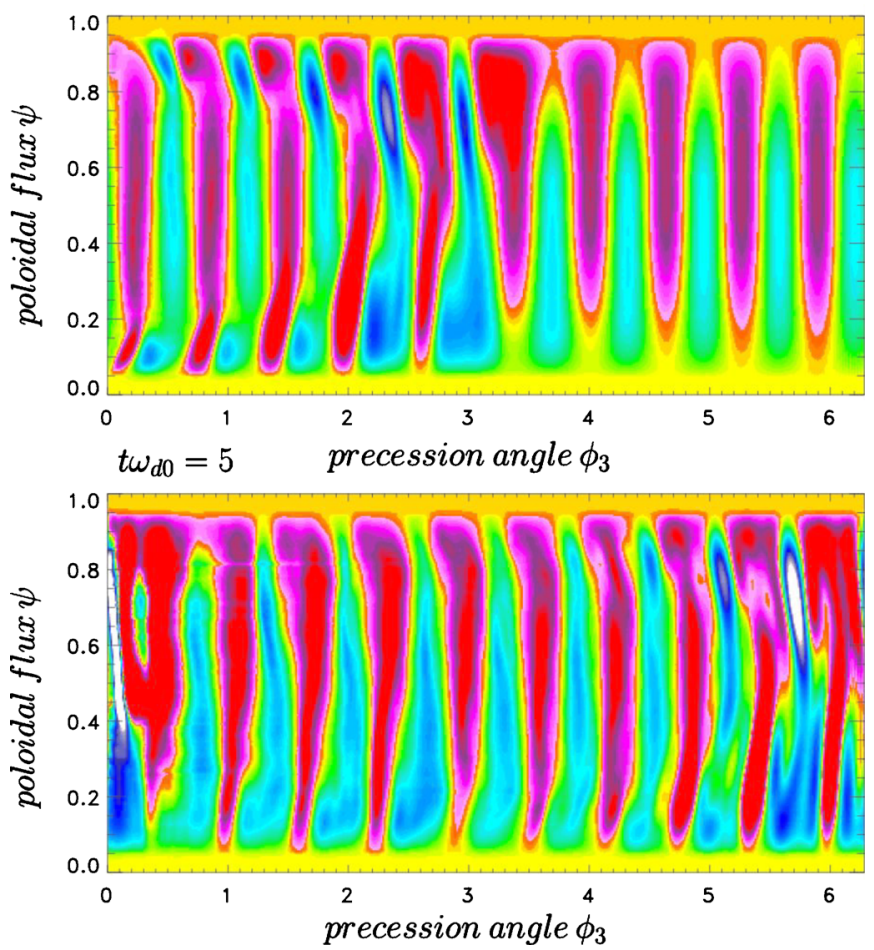

FIG. 5. (Color online) Phase space representation of the electric potential during the initial phase of the ITG instability, for a value of the magnetic shear of $s=0.5$. Streamerlike structure is observed corresponding to the formation of ten filaments in agreement with the initial perturbed mode $n=10$.

tom panel), the dominant modes are $n=10$ and $n=30$, as the result of the occurring of nonlinear mode coupling. Finally at time $t \omega_{d 0}=40$, the nonlinear coupling disappears and the system returns to the initial state, now dominated by the streamer's growth on the mode $n=10$. The mechanism responsible for such mode coupling is not well understood although exactly resonant three-wave interactions have been implicated: The spectrum in frequency broadens and shows the possibility of triad interactions of the type $n_{3}=n_{2}+n_{1}$ and $\omega_{3}=\omega_{2}$ $+\omega_{1}$, usually met in the Kolmogorov cascade. ${ }^{37}$ However it must be pointed out that the standard Kolmogorov cascade usually met in fluid turbulence cannot be applied here since only a restricted number of modes is excited in our system. The mechanism of the instability is induced by wave-particle resonance at the precessional frequency $\omega_{d}$. Here in the gyrokinetic Vlasov approach, the energy is distributed along the adiabatic invariants $\mu$ (or equivalently the pitch-angle parameter $\kappa$ ) and the energy $E$, allowing particle-wave resonances.

The instability starts up without any polarization term, usually necessary in the HM model. The corresponding electric potential behavior is shown in phase space in Figs. 5-7 for the three phases of the instability for $N_{E}=128$. The corresponding time evolution of the potential mode $n=10$ is thus shown in the bottom panel in Fig. 3. The linear phase (estimated in the range $0 \leq t \omega_{d 0} \leq 7$ ) exhibits the growth of the expected mode $n=10$, which is here characterized by the formation of a streamerlike structure, elongated along the $\psi$ coordinate. Here the TIM system (for a magnetic shear of
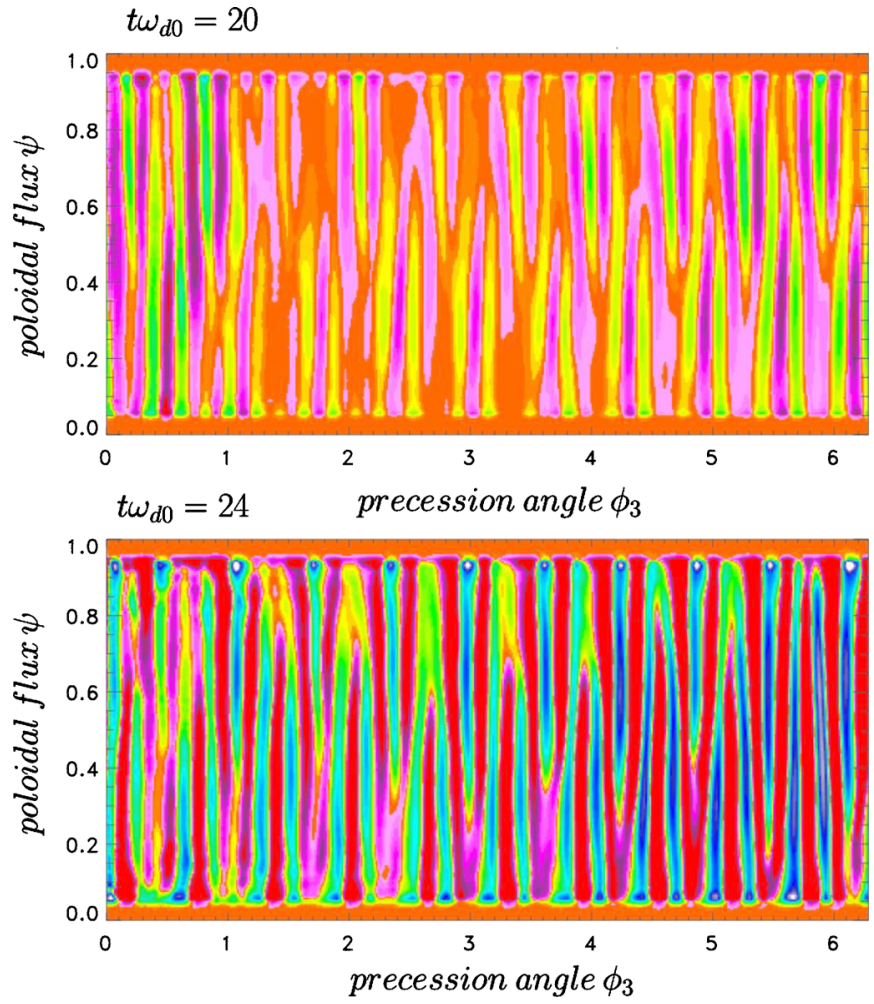

FIG. 6. (Color online) Continuing the presentation of the behavior of the electric potential in the second phase of the ITG instability: We observe now the nonlinear mode coupling with the appearance and strong growth on the second mode $n=30$.
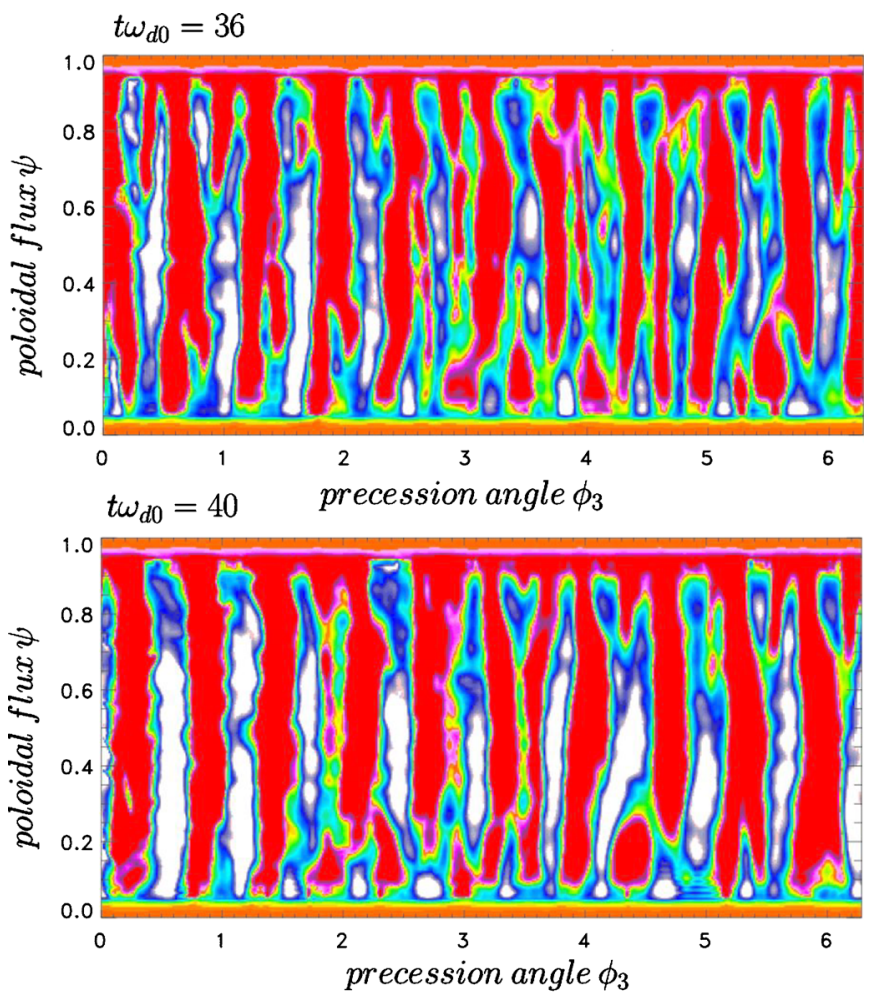

FIG. 7. (Color online) The corresponding phase space representation at later times during the saturation phase. The simulation was performed with $s$ $=0.5$. We observe a more complex behavior characterized by the damping of the second dominant mode and ten streamers are now apparent. 
$s=0.5)$ allows a dominant role of streamers due to the relative weakness of zonal flows. The forward energy transfer is carried out by three-wave interactions that couple the initially perturbed mode (the streamer mode $n_{1}=10$ ) to its harmonics $n_{2}=20$ and $n_{3}=30$. The nonlinearity of the distribution $f_{E, \kappa}$, induced by the $\mathbf{E} \times \mathbf{B}$ drift term, yields to the growth of the dominant mode $n_{3}=30$. At time $t \omega_{d 0}=24$ in Fig. 6, the phase space behavior indicates clearly the occurring of 30 streamerlike structures. In that regime, zonal flows have practically no impact on the saturated transport level, implying that the dominant saturation mechanism must be of a different nature. In Fig. 7, well inside the saturation regime, we see clearly that only ten streamers are now present in the electric potential. A possible explanation is that the threewave interaction between TIMs ceases (like by detuning process). Such three-wave interaction mechanism was also predicted by Terry et al. ${ }^{24,38}$ for TEM in the case of a two-field model (density- potential) allowing an inverse cascade process induced by the nonlinearity in the potential equation. Here the situation is however a little more complex since wave-particle resonances lead to a kinetic-type dissipation and the density and the electric potential can remain in phase in the process.

\section{B. Polarization effect}

The polarization effect appears in the Poisson equation or here in the quasineutrality condition (25) by the introduction of the polarization density (the Laplacian term). It is due to the difference between the real trapped particle density and the bounce-averaged banana center density. Densities are defined from the distribution function of particles $f$ while the bounce-averaged gyrokinetic equation (22) [with the data of the characteristics (23) and (24)] deals with the smoothed (bounce plus cyclotron averaged) $\bar{f}_{E, \kappa}$ distribution function. The resulting density fluctuation $\bar{n}_{i}$, for banana centers, can be related to $\bar{f}_{E, \kappa}$ in the following way: ${ }^{39}$

$$
\begin{aligned}
\bar{n}_{i}= & \frac{2}{\sqrt{\pi}} \int_{0}^{\infty} d E \sqrt{E} \int_{0}^{\kappa_{\max }} d \kappa \kappa K(\kappa) \hat{J}_{0} \bar{f}_{\kappa, E}-n_{0} \\
& -\frac{2}{\sqrt{\pi}} \int_{0}^{\infty} d E \sqrt{E} \int_{0}^{\kappa_{\max }} d \kappa \kappa K(\kappa)\left(1-\hat{J}_{0}^{2}\right) f_{\mathrm{eq}} \tilde{U},
\end{aligned}
$$

where the equilibrium distribution function is Maxwellian $f_{\text {eq }}=n_{0} e^{-E}$ (keeping the normalization of the energy to $T_{0}$ ). Once projected onto the Fourier base, the last integral in Eq. (67) is proportional to $1-J_{0}^{2}(\xi \sqrt{E})$ with $\xi=n \rho_{c i}+k_{\psi} \delta_{b}(\kappa)$ (where $\rho_{c i}$ and $\delta_{b}$ are normalized to $\Delta \psi$ ). This quantity may be approximated by the following equation:

$$
\begin{aligned}
& \frac{2}{\sqrt{\pi}} \int_{0}^{\infty} d E \sqrt{E} \int_{0}^{\kappa_{\max }} d \kappa \kappa K(\kappa)\left[1-J_{0}^{2}(\xi \sqrt{E})\right] e^{-E} \\
& \quad \simeq 1-I_{0}\left(\xi^{2}\right) e^{-\xi^{2}}
\end{aligned}
$$

using the approximation $\delta_{b}(\kappa)=$ const. Here $I_{0}$ is the modified Bessel function of the first kind. For the sake of simplicity, we keep only the first term $\xi^{2}$ in the expansion $\xi \rightarrow 0$. In the real space, this term is then equivalent to the standard

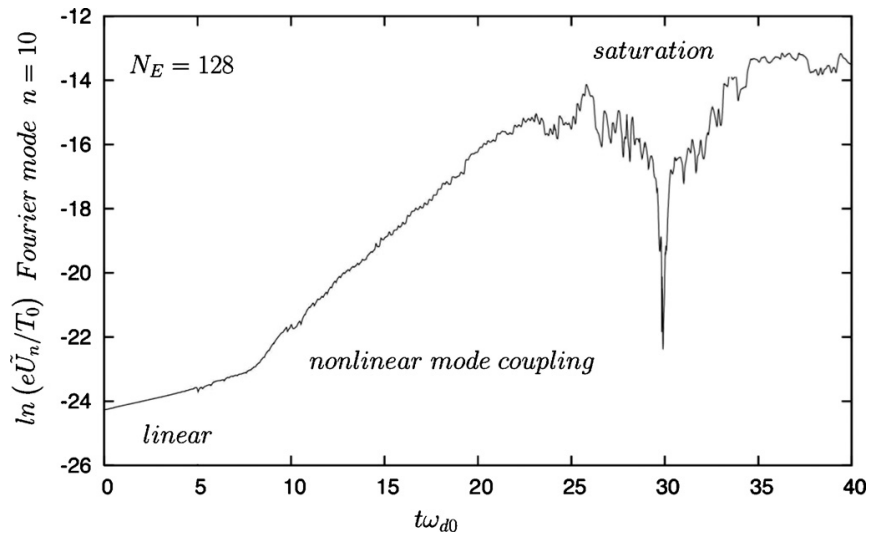

FIG. 8. Time evolution of the Fourier mode $n=10$ of the electric potential fluctuations performed when the polarization density is taken into account. The simulation parameters are identical to those of the plot of Fig. 3 without polarization. The simulation parameters are $\Delta \tau-\Delta \tau_{s}=0.0075, \Delta \tau_{s}=0.1075$, and a magnetic shear of $s=0.5$. Three different phases may be observed: a linear one followed by the nonlinear mode coupling and finally the phase of saturation.

form of the normalized operator $\xi^{2} \rightarrow \overline{\nabla^{2}} \equiv \rho_{c i}^{2} \partial_{\phi_{3}}^{2}+\delta_{b}^{2} \partial_{\psi}^{2}$ where $\delta_{b} \simeq q_{0} \rho_{c i} / \sqrt{\varepsilon} \Delta \psi$ and $\rho_{c i}=r_{0} \sqrt{m_{i} T_{0}} / e q_{0}$.

Recently the formalism of one-form mechanics and Lieperturbation theory ${ }^{20}$ was employed. In particular, the full banana orbit width effect was retained in both bounceaveraged Vlasov and Poisson equation. Our analysis differs from the general form of the neoclassical polarization density given by Fong and $\mathrm{Hahm}^{20}$ in the sense where the authors take into account the deviation from the bounce center radial $(\psi)$ and field line $\left(\phi_{3}\right)$ position. Here we have assumed that the quantity $\varphi_{G}=\phi_{3}+q\left(r_{0}\right) \hat{\theta}\left(\boldsymbol{J}, \phi_{2}\right)+\hat{\varphi}\left(\boldsymbol{J}, \phi_{2}\right)$ given in Eq. (9) is approximated to $\phi_{3}+q\left(r_{0}\right) \hat{\theta}(\phi)$, i.e., neglecting the deviation from the regular precession motion $\hat{\varphi}$. Recently ${ }^{39}$ we have shown that the electron response (in particular, in an adiabatic form) may modify the nature of ion turbulence. The electron response to zonal flow modes was found to govern the characteristics of the convective cells in the turbulent regime, and streamer $\left(\varepsilon_{e}=0\right)$ or zonal flows $\left(\varepsilon_{e}=1\right)$ dominate depending on whether the electron response is adiabatic or zero.

To check this feature, we have performed a numerical simulation for an ITG threshold of $\Delta \tau_{s}=0.1075, \Delta \tau-\Delta \tau_{s}$ $=0.0075$ being identical to the case shown in Fig. 3 (bottom panel), a magnetic shear of $s=0.5$, and a banana width of $\delta_{b}=0.010 \Delta \psi$. We have used a phase space sampling of $N_{\psi} N_{\phi_{3}}$ of $128 \times 512$ points and a number of adiabatic invariants of $N_{E} N_{\kappa}$ of $128 \times 16$. We use a time step of $\Delta t \omega_{d 0}$ $=0.005$ and $C_{e}=0.10$. We keep the case $\varepsilon_{e}=0$ to study the streamer regime of the TIM instability. Numerical results are shown in Figs. 8 and 9 and the corresponding phase space representation of the electric potential in Fig. 10. Figure 8 shows, on a logarithmic scale, the time evolution of the electric potential mode $n=10$, when both polarization effects and electron adiabatic response are taken into account. The temporal behavior is somewhat similar to the previous case, without polarization and when $\varepsilon_{e}=0$ : The linear phase presents the same growthrate $\gamma_{\text {lin }} \simeq 0.06 \omega_{d 0}$ and the mode cou- 

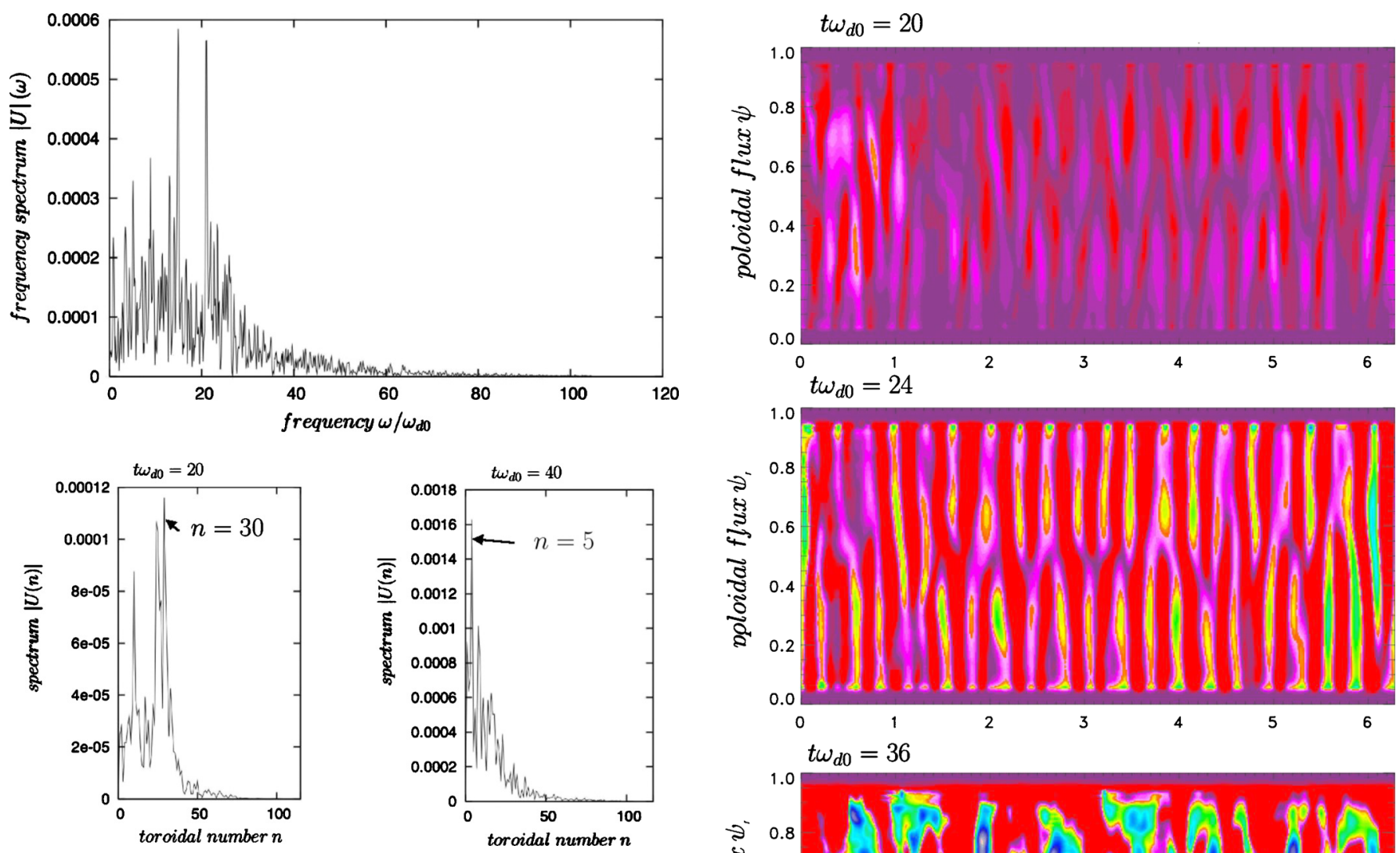

FIG. 9. Corresponding frequency spectrum on the top panel and the wavenumber spectrum at two different times during the simulation on bottom panels. Here the polarization density is included. The magnetic shear is $s=0.5$.

pling leads to the growth of several modes. In particular, the mode $n=10$ exhibits again the same growthrate $\delta_{n l}$ $\simeq 0.25 \omega_{d 0}$. One notes however a strong reduction of the saturation level of the amplitude of the electric potential in this regime and the corresponding frequency spectrum of the quantity $e \tilde{U} / T_{0}$ differs now showing that the saturation mechanism is modified. The growth of the ITG instability is balanced by the occurring of a larger-scale structures as LSSS modes, as can be seen in the bottom right panel in Fig. 9. Usually due to their fundamentally different character, the coexistence of both types (streamers or zonal flows) can be excluded; rather, one (or none) of them will dominate. Figure 10 shows the behavior of the electric potential fluctuations in phase space. Small structures appear as a consequence of the nonlinear mode coupling at time $t \omega_{d 0}=20$. At time $t \omega_{d 0}=24$ several Fourier modes are now present and close to the dominant mode $n=21$. These structures seem to coalesce leading to a saturated state dominated by larger-scale structures. In particular we expect to the presence of the mode $n=0$ a zonal flow (structure elongated along $\phi_{3}$ ). However the zonal flow seems to remain at a very weak level in this studied regime, where streamers are also present. In the following sections, we will address the questions of the occurring of dominant zonal flow (in particular, in the saturation process) for different values of the magnetic shear.
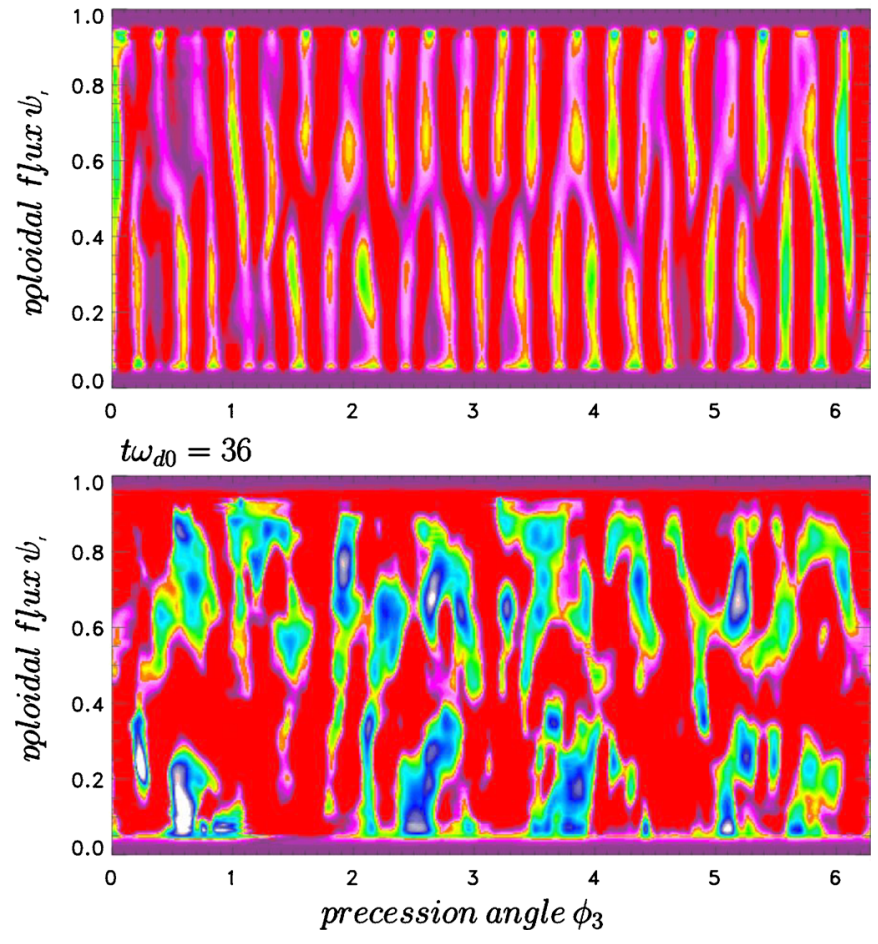

FIG. 10. (Color online) Corresponding behavior of the potential fluctuations in the $\left(\psi, \phi_{3}\right)$ space: The saturated state is now dominated by large-scale streamer-type structures. We do not observe the presence of a zonal flow $n=0$. Here the magnetic shear is $s=0.5$ and the polarization density is taken into account.

\section{TIM turbulence for high values of the magnetic shear}

The next simulation is performed with a magnetic shear of $s=2.5$ well inside the strong regime of the fully developed turbulence, the other physical parameters being unchanged. Figure 11 shows the time evolution of two different Fourier modes of the electric potential fluctuations $n_{1}=10$ and $n_{2}$ $=31$ (on top and bottom panels in Fig. 11, respectively). The corresponding frequency spectrum is plotted on top panel in Fig. 12 together with the spectra as a function of $n$ (toroidal number) at two different times $t \omega_{d 0}=12$ (bottom left panel) and $t \omega_{d 0}=30$ (bottom right panel). The first time corresponds to an intermediate state whereas the second is taken at the saturation of the instability. The spectrum in frequency shows a number of fluctuation peaks. The peaks satisfy the linear dispersion relationship given by Eq. (43). Many of the peaks are excited by nonlinear couplings with others peaks. 

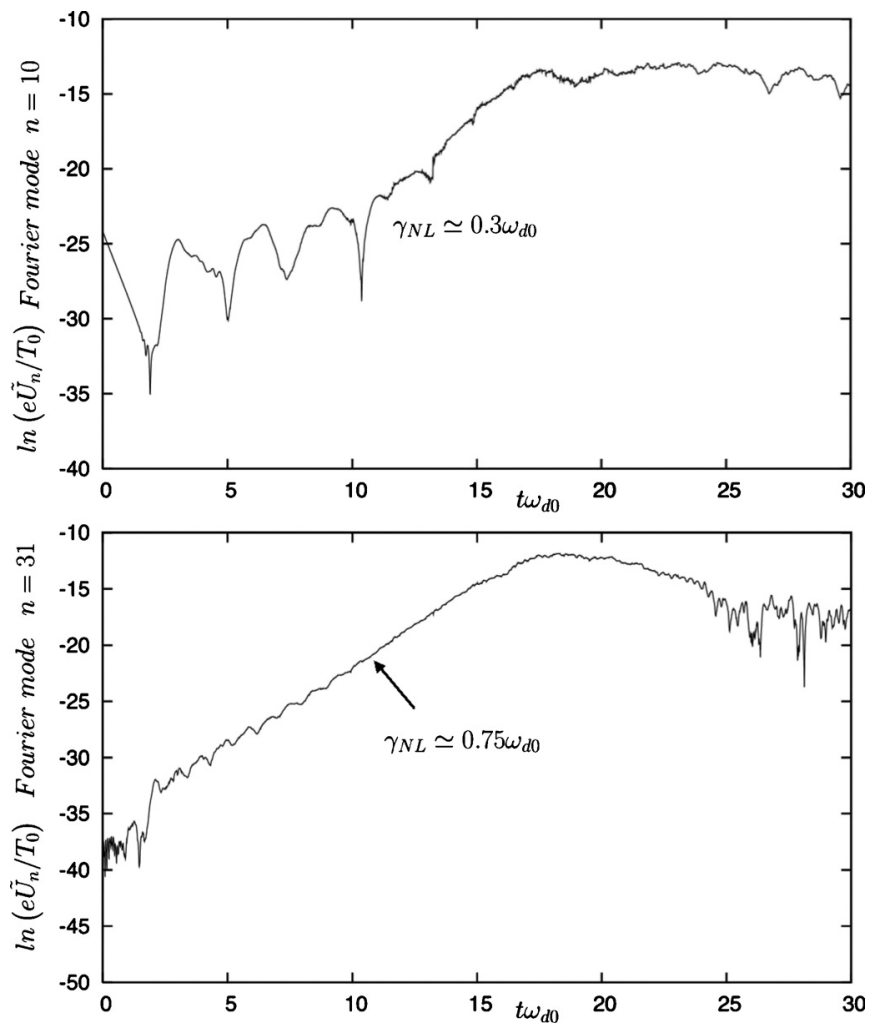

FIG. 11. Now carried out with a magnetic shear of $s=2.5$, on the top panel the time evolution of the mode $n=10$ and on the bottom panel, the behavior of the mode $n=31$ (shown in a logarithmic scale).

We observe that the mechanism of turbulence trigger is due to the three-wave interaction processes. In particular, we observe the parametriclike nonlinear coupling $\omega_{3}=\omega_{1}+\omega_{2}$ and $n_{3}=n_{1}+n_{2}$ (the corresponding frequencies were indicated on the top panel). Table I gives the frequencies and toroidal numbers of the different TIMs. We observe here that the intermediate mode $\left(n_{2}, \omega_{2}\right)$ exhibits a dominant peak close to the value predicted by the linear theory $\omega_{2}=\frac{3}{2} n_{2}\left\langle\omega_{d}\right\rangle_{\kappa}$ $\simeq 116.2 \omega_{d 0}$ using a mean value of $\left\langle\omega_{d}\right\rangle_{\kappa} \simeq 2.5$ (for a magnetic shear of $s=2.5$ ) and a wavevector of $n_{2}=31$. (This mode was plotted at the bottom panel in Fig. 11, the mode $n_{3}=41$ is not represented here; however we have observed in the simulation the growth of more 50 modes in time).

Some features of the instability must be pointed out.

First, we have identified the streamer structure $\left(n_{3}\right.$ $=41$ ) in the phase space in the growth phase (i.e., the nonlinear coupling regime of TIMs) as can be observed in Fig. 13, where we have plotted the electric fluctuating potential at three different times. First at time $t \omega_{d 0}=15$ we observe the beginning of the occurring of streamers: We may observe 41 structures which give a clear indication that it is effectively the mode $n_{3}$ which is here dominant. At later time $t \omega_{d 0}$ $=18$ this mode reaches its maximum value and the streamer structure was shown to be extended in the $\psi$ direction (radial direction). The growth of the streamer mode continues until a suppression mechanism sets on. The time evolution indicates an inverse energy cascade from the short scale structure toward
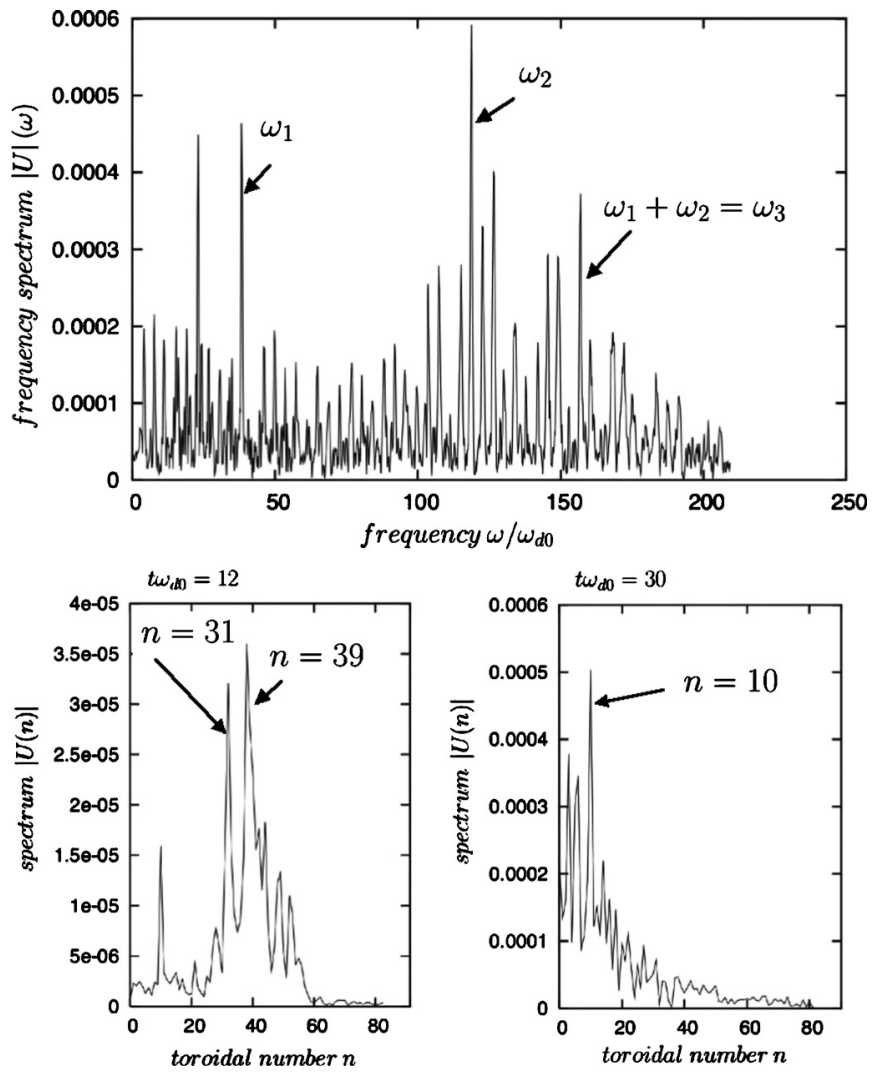

FIG. 12. Corresponding plot in frequency spectrum on the top panel together with the spectra in wave-numbers (here as a function of the toroidal number $n$ ) at two different times on the bottom panels. The simulation and physical parameters correspond to those of Fig. 11 (magnetic shear of $s$ $=2.5$ ).

larger scales. The nonlinear triplets coupling (i.e., according to $n_{3}=n_{1}+n_{2}$ ), that leads to streamer formation, determines here multiple characteristic time scales and (mesoscopic) spatial scales in turbulent plasmas.

(ii) Spatial spectrum analysis of interchangelike TIM turbulence in this simulation clearly displays a condensation tendency of turbulent fluctuation energy toward a finite mode $(n \neq 0)$, as typically shown at the bottom right panel in Fig. 12. Note that the frequency of the mode $n=6$ is clearly visible in the upper pattern of the frequency spectrum: We expect the linear value $\omega$ $=\frac{3}{2} n\left\langle\omega_{d}\right\rangle_{\kappa} \simeq 22.5 \omega_{d 0}$ (close to the numerical value of $\omega_{\text {num }} \simeq 22.10 \omega_{d 0}$ whereas the zonal flow mode remains at a weak level but not however negligible). The saturated state which follows is characterized by

TABLE I. Corresponding values of frequencies obtained by solving the linear dispersion relation compared with the numerical values obtained by the SL Vlasov code for the TIM instability.

\begin{tabular}{lccc}
\hline \hline Mode & $n$ & Frequency $\omega=\frac{3}{2} n\left\langle\omega_{d}\right\rangle_{\kappa}$ & (in $\omega_{d 0}$ units) \\
\hline$n_{1}$ & 10 & 37.5 & 38.0 \\
$n_{2}$ & 31 & 116.2 & 117.0 \\
$n_{3}$ & 41 & 153.7 & 154.0 \\
\hline \hline
\end{tabular}



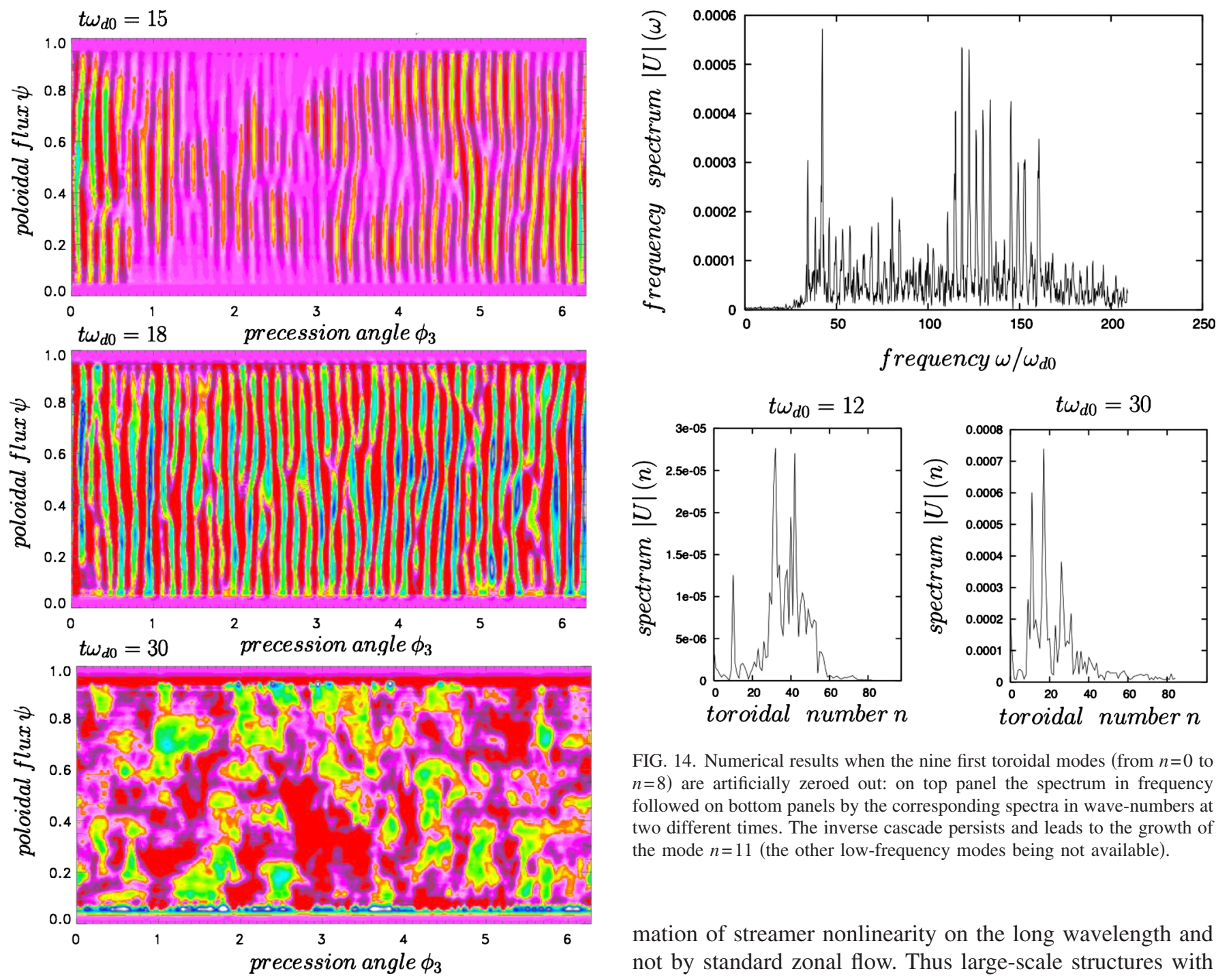

FIG. 14. Numerical results when the nine first toroidal modes (from $n=0$ to $n=8)$ are artificially zeroed out: on top panel the spectrum in frequency followed on bottom panels by the corresponding spectra in wave-numbers at two different times. The inverse cascade persists and leads to the growth of the mode $n=11$ (the other low-frequency modes being not available).

FIG. 13. (Color online) Fluctuating electric potential representation in the $\left(\psi, \phi_{3}\right)$ space, for a value of the magnetic shear of $s=2.5$, continuing the presentation of results of Figs. 11 and 12 for the same simulation.

the domination of larger-scale TIM (here the mode $n=6$ ) induced by the inverse energy cascade. Such an inverse energy cascade has been observed experimentally $^{40}$ with the observation of several matching rules for frequencies (for three-wave interactions), broad turbulence spectra including spectral condensation and zonal flow generation.

\section{Inverse cascade toward LSSS}

We report here the numerical evidence of the inverse energy cascade which results in the generation of the broadband spectrum and large-scale (TIM) turbulent structures, but which differ from the standard zonal flow usually met in the saturation process of ITG in toroidal plasmas. From a physical point of view turbulent transport depends on both (spatial) spectrum nature and fluctuation level, which are represented by effective nonlinear decorrelation length and time decorrelation. In contrast to the ITG standard case TIM turbulence may be saturated by inverse cascade with the for-

mation of streamer nonlinearity on the long wavelength and not by standard zonal flow. Thus large-scale structures with low frequencies may interact with the primary fluctuations and play a key role in the TIM turbulence saturation. Here the dynamics of large-scale structures, generated by inverse cascade, may become a mediator in the spectral cascading and saturate TIM turbulence in Vlasov simulations.

We would like to assess here the role of such LSSS modes and the inverse cascade in the collisionless TIM turbulence. To this end, we compare "normal" TIM simulations to run in which the nine first modes (from $n=0$ to $n=8$ ) are artificially zeroed out (and, in particular, the zonal flow $n$ $=0$ is suppressed forbidding any growth). Figure 14 shows the corresponding numerical results of the simulation, performed with the same physical parameters used in Figs. 11-13, except that we have suppressed the nine first modes. (A first simulation performed with the artificial suppression of modes $n=0$ until $n=2$ exhibits indeed exactly the same features, implying that zonal flows are not the dominant saturation mechanism here.) A very different behavior can be observed when we choose to remove only the large-scale dynamics in nonlinear interaction (characterized by the modes from $n=4$ to $n=6$ ). The simulation shows that the inverse cascade persists and the low $n$-quasimodes are formed in the frequency spectrum (up panel in Fig. 14 and in the spatial spectrum at $t \omega_{d 0}=30$ in the asymptotic state at bottom right panel). Indeed the only path toward the conden- 
sation is now possible through the excitation of the modes $n>9$. Although the beginning of the plasma evolution remains identical in comparison with the case where we have kept all modes (as can be seen at time $t \omega_{d 0}=12$ at the bottom left panel in Fig. 14), the asymptotic state differs since we have forbidden an inverse cascade process till the lower mode $n=4$. The system evolves until a saturation state of $n$ $=12$ streamerlike structure at time $t \omega_{d 0}=30$ (not shown here). These observations show that there may exist a correlation between the excitation of long-wavelength LSSS structures and the TIM turbulence saturation. Thus one of the prominent saturation mechanisms is the inverse energy cascade, which originates from nonlocal mode coupling processes in TIM turbulence. The general nonlinear coupling (here the toroidal coupling due to the curvature of the magnetic field) is a key process to excite the large wavelength quasimode, which in turn have a backreaction on the TIM turbulence saturation.

\section{E. Temperature gradient threshold influence}

We now investigate the TIM instability as a function of $\Delta \tau$. A very different behavior can be observed for reasonably large ion temperature gradients. We first check the existence of a threshold in the TIM turbulence as predicted by the model. The ITG threshold, for the case of TIM, is given by formula (49), which indicates, in particular, that for usual tokamak plasma parameters, the ITG becomes stable if the temperature gradient is chosen lower than $\Delta \tau_{s}$. We have checked such a property by performing a simulation with a value of the temperature gradient of $\Delta \tau=0.08$, chosen well below the theoretical threshold of $\Delta \tau_{s}=0.100075$ and with the same value of the magnetic shear $s=2.5$. The simulation results, not shown here, confirm that the system remains stable.

In the previous subsection, we have analyzed the nonlinear dynamics of the system near the ITG instability threshold: The dynamics is dominated by the forward cascade in energy followed by the condensation process in the saturation mechanism. Such a mechanism involves three-wave parametriclike interaction and may be considered as a critical nonlinear phenomenon for kinetic instability near the threshold of the instability. It is a competition process with the standard linear growth of the interchangelike instability. In particular, if we choose to increase the gradient of the ITG instability, i.e., by increasing the parameter $\Delta \tau$ we may expect that the interchange instability becomes the dominant process with a stronger linear growthrate.

Results of a simulation carried out with a larger value of the temperature gradient of $\Delta \tau=0.12$ are shown in Fig. 15, the magnetic shear being kept to $s=2.5$, i.e., well inside the streamer regime. The frequency spectrum (shown in the top panel in Fig. 15) indicates clearly that the initial mode perturbation (here $n=10$ ) remains the dominant mode, even in the asymptotic regime of the saturation although the two first harmonics are also present in the spectrum in wavevector but remain at a very weak level (see the bottom left panel in Fig. 15 at time $\left.t \omega_{d 0}=10\right)$. The dominant peak is observed at the numerical frequency $\omega \simeq 41.7 \omega_{d 0}$, which is somewhat higher
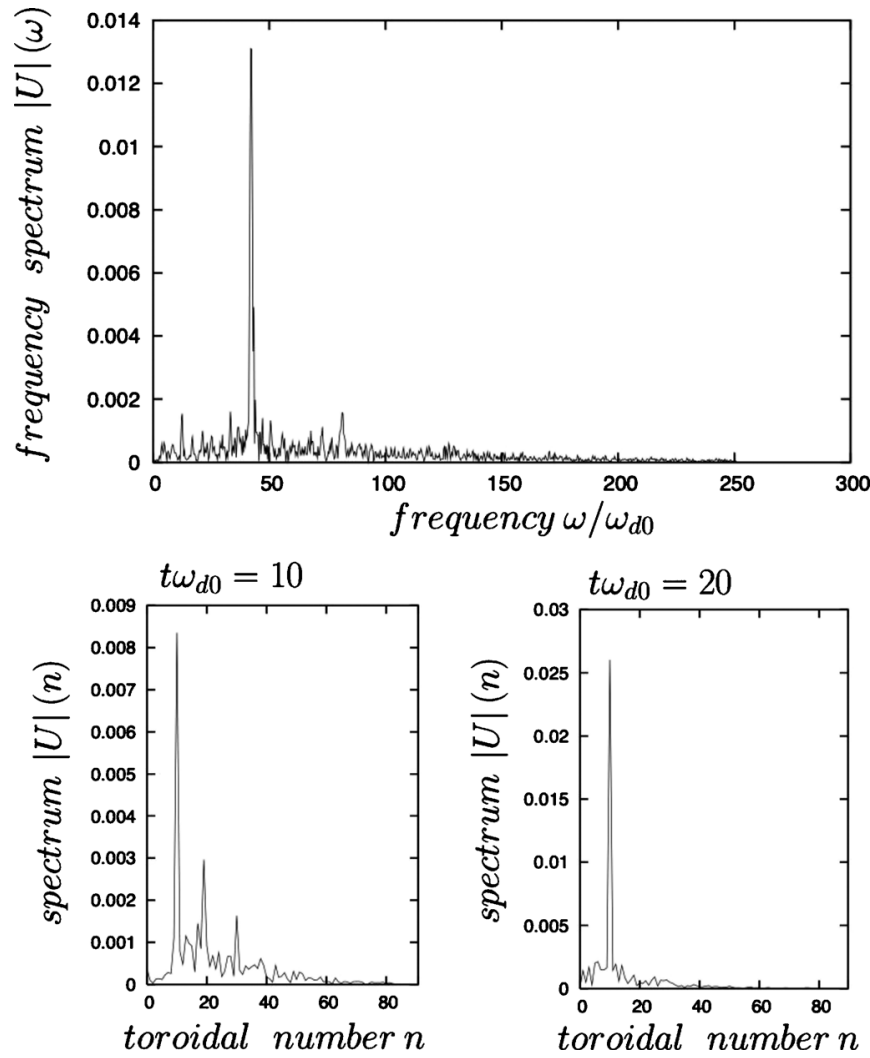

FIG. 15. Numerical results concerning a simulation carried out with a higher ion temperature gradient of $\Delta \tau=0.12$, well above the threshold of $\Delta \tau_{s}$ $=0.100075$. At time $t \omega_{d 0}=20$, in the phase of the instability's saturation, only the mode $n=10$ is present.

than the expected linear value of $\omega=\frac{3}{2} n\left\langle\omega_{d}\right\rangle_{\kappa} \simeq 37.5 \omega_{d 0}$ using $\left\langle\omega_{d}\right\rangle_{\kappa} \simeq 2.5$, which is probably due to the high value of the growthrate of the instability and of the presence of several harmonics. The temporal evolution and the suppression of the triad interaction and the inverse energy cascade lead to the development of a saturated state with clear indication of the formation of a streamer structure on the mode $n=10$. The nature of the final state depends crucially on the value of the ion temperature gradient. The nonlinear phase of the evolution of the TIM instability is characterized by the growth of the linearly unstable mode (here only the mode $n=10$ is perturbed initially) and the occurrence of the expected nonlinear mode coupling. In the saturated state, which follows the suppression of the nonlinear mode coupling, the initially perturbed mode $n=10$ then becomes the dominant mode and the spatial spectrum, shown at the bottom right panel in Fig. 15, exhibits clearly only the corresponding peak. This occurs because the smaller scale instabilities that support the growth of triad excitation got suppressed by the most unstable instability, here the interchangelike ITG instability.

\section{F. Transport scaling}

The thermal diffusivity is now studied in this section and we show that it exhibits a Bohm-like scaling. Bohm-like scaling may be expected and is relevant to several scenarios, including turbulence spreading ${ }^{41-43}$ from the linearly active (unstable) region to the linearly inactive (stable) region. The 
standard approach involves a random walk type of picture for diffusive processes using the scale length of streamer structure as the step size (close to the system size $\Delta \psi$ when streamers dominates) and a time correlation of $\omega_{d 0}^{-1}$ as the time step. The transport scaling is Bohm-like with a thermal diffusivity proportional to $\chi_{B}=\omega_{d 0} \Delta \psi^{2}=T_{0} / e B_{0}$. On the other hand, in the gyro-Bohm scaling, we expect a correlation length of $\sqrt{\delta_{b} \Delta \psi}$. The thermal diffusivity is then $\chi_{g B}$ $=\chi_{B} \delta_{b} / \Delta \psi$. Thus the thermal diffusivity may be estimated by the relation

$$
\frac{\chi}{\chi_{B}}=\left(\frac{\delta_{b}}{\Delta \psi}\right)^{\alpha},
$$

where $\alpha=1$ for the gyro-Bohm scaling and $\alpha=0$ for the standard Bohm scaling. To check this point five numerical simulations have been performed for different values of the parameter $\delta_{b} / \Delta \psi$.

Averaging the gyrokinetic Vlasov equation (22) using characteristics (23) and (24) leads to the heat equation

$$
\frac{3}{2} \frac{\partial P}{\partial t}+\frac{\partial Q}{\partial \psi}=0,
$$

where the pressure term is given by

$$
\frac{3}{2} P(\psi, t)=\int_{0}^{2 \pi} \frac{d \phi_{3}}{2 \pi}\left\langle E f_{E, \kappa}\right\rangle_{\kappa, E} .
$$

The corresponding heat flux is then

$$
Q=\int_{0}^{2 \pi} \frac{d \phi_{3}}{2 \pi}\left\langle E\left(-\frac{\partial}{\partial \varphi_{3}}\left(\hat{J}_{0} \tilde{U}\right)\right) f_{E, \kappa}\right\rangle_{\kappa, E},
$$

where the mean operator denotes the following quantity:

$$
\langle.\rangle_{\kappa, E}=\int_{0}^{1} d \kappa \kappa K(\kappa) \frac{2}{\sqrt{\pi}} \int_{0}^{+\infty} d E \sqrt{E} .
$$

In order to obtain an estimation of the thermal diffusivity ratio $\chi^{\prime} \chi_{B}$, we compare the computed turbulent flux given by the semi-Lagrangian Vlasov solver with the quasilinear value using Eq. (72). The time-averaged heat flux defined above can be written using Fourier series as

$$
Q(\psi)=\sum_{n} \int_{-\infty}^{+\infty} \frac{d \omega}{2 \pi}\left\langle i n \hat{J}_{0} \widetilde{U}_{n \omega}^{*}(\psi) f_{E, \kappa, n \omega}\right\rangle_{\kappa, E} .
$$

Using the linear expression of $f_{E, \kappa, n \omega}(\psi)$ used in Sec. IV to establish the linear dispersion relation for TIM, we then obtain

$$
\begin{aligned}
Q_{Q L}= & -2 \sqrt{\pi} \Delta \tau \sum \int_{0}^{1} d \kappa \kappa K(\kappa)_{n} \frac{1}{2 \pi} \\
& \times \int_{-\infty}^{+\infty} \frac{d \omega}{n \omega_{d}(\kappa)}\left|\hat{J}_{0} \widetilde{U}_{n \omega}\right|^{2} \Theta_{n}(\omega, \kappa),
\end{aligned}
$$

where we have defined $\Theta_{n}$ by

$$
\Theta_{n}=\left(\frac{\omega}{n \omega_{d}(\kappa)}\right)^{3 / 2} \exp \left(-\frac{\omega}{n \omega_{d}(\kappa)}\right)\left(\frac{\omega}{n \omega_{d}(\kappa)}-\frac{3}{2}\right) .
$$

Finally we obtain (using $\chi_{B}=\omega_{d 0} \Delta \psi^{2}$ ) the relation
TABLE II. Values of the mean quasilinear turbulent heat flux $e Q_{Q L} / T_{0}$, the numerical value $e Q / T_{0}$, and the thermal diffusivity normalized to the Bohm value $\chi^{\prime} \chi_{B}$ for different values of the banana width normalized to the system length.

\begin{tabular}{lccc}
\hline \hline$\delta_{b} / \Delta \psi$ & $e Q_{Q L} / T_{0}$ & $e Q / T_{0}$ & $\chi / \chi_{B}$ \\
\hline 0.010 & $-6.53 \times 10^{-5}$ & $-4.50 \times 10^{-5}$ & 0.69 \\
0.025 & $-0.99 \times 10^{-6}$ & $-0.75 \times 10^{-6}$ & 0.75 \\
0.050 & $-2.30 \times 10^{-7}$ & $-1.75 \times 10^{-7}$ & 0.76 \\
0.075 & $-3.95 \times 10^{-9}$ & $-3.0 \times 10^{-9}$ & 0.75 \\
0.1 & $-2.02 \times 10^{-11}$ & $-1.5 \times 10^{-11}$ & 0.75 \\
\hline \hline
\end{tabular}

$$
\frac{\chi}{\chi_{B}}=\frac{Q}{Q_{Q L}} .
$$

Numerical results are presented in Table II for different values of the ratio of the banana width over the system length $\delta_{b} / \Delta \psi$. Simulations were carried out with the following physical parameters $\Delta \tau=0.12$ and a magnetic shear of $s=2.5$ (the corresponding simulation for $\delta_{b} / \Delta \psi=0.01$ is shown in Fig. 15 and corresponds to the limit of the streamer regime since the spectrum exhibits only a single frequency). However when we increase $\delta_{b} / \Delta \psi$, the interchange instability decreases and the system enters into the streamer regime. Numerical estimation of the thermal diffusivity has been obtained in the linear phase of the instability before the forward cascade takes place. In Table II we give the numerical value of the heat flux $e Q / T_{0}$ averaged over the poloidal flux $\psi$. We see clearly that this quantity is much lower than the quasilinear estimate (close to $75 \%$ ) but remains constant as a function of the ratio $\delta_{b} / \Delta \psi$, which indicates a Bohm-like scaling during the first phase of the instability (streamer growth). During the nonlinear saturation of the instability the situation is somewhat more complicated since an accurate estimate of the quasilinear heat flux given by Eq. (75) requires a lot of modes.

\section{G. Low magnetic shear regime}

We analyze now the effect of the magnetic shear which plays a major role in determining the generation of streamers (for high values of $s$ ) or zonal flows in a domain of low magnetic shear values. Such parametric dependence in the high magnetic shear window is favorable for streamer growth. On the contrary we expect a stabilizing mechanism for TIM instability for a negative magnetic shear. However this process is accompanied by the generation of a zonal flow. To confirm this point a numerical simulation with a negative magnetic shear of $s=-0.5$ is performed. We have used a temperature gradient $\Delta \tau$ just above the threshold of the ITG instability (with $\Delta \tau-\Delta \tau_{s}=0.0075$ ). The introduction of a reversal shear leads to a complete stabilization of the ITG-driven ion trapped instability. The initially excited TIM (on the mode $n=10$ ) damps and remains at a very weak level during all the simulation. The corresponding spectra are 

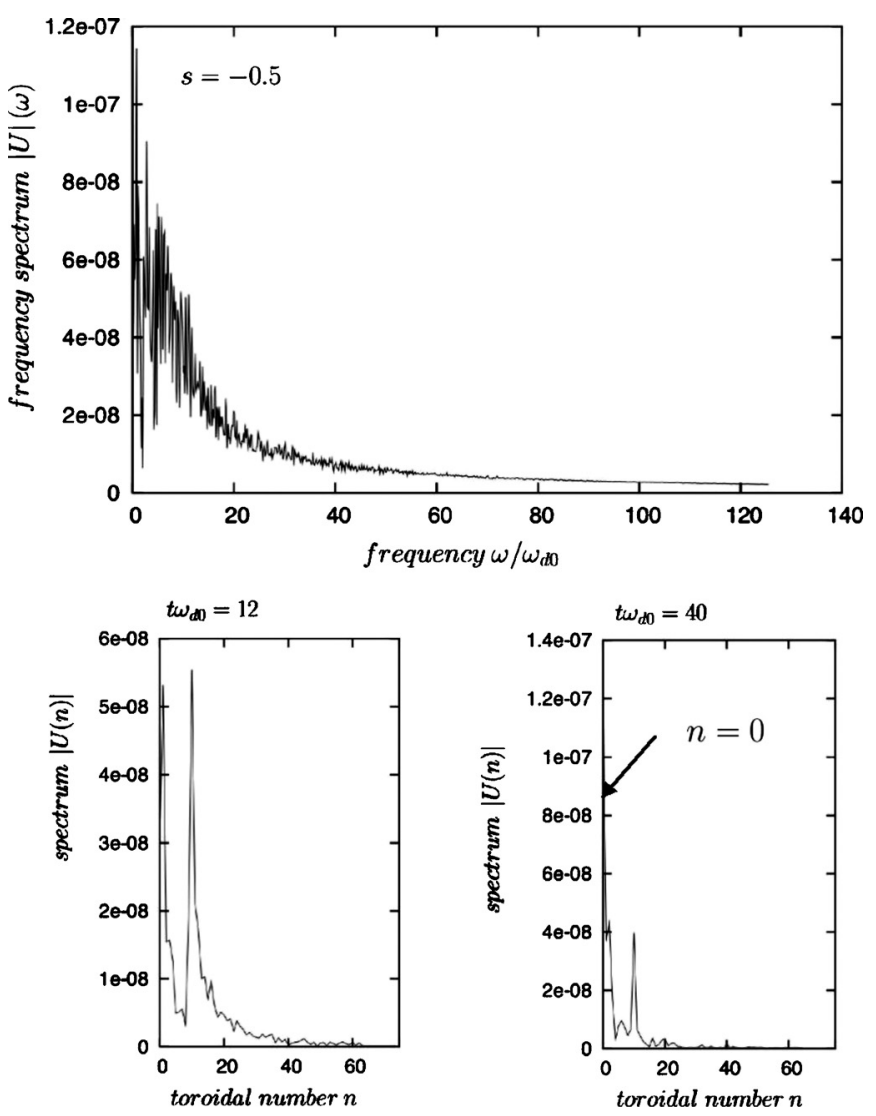

FIG. 16. The case with a negative magnetic shear of $s=-0.5$ : on top panel the spectrum in frequency of the electric potential followed on bottom panels by the spectra in wave-numbers of the same quantity. Note now the presence of a zonal flow which characterizes the stabilization process.

shown in Fig. 16: The top panel exhibits the spectrum in frequency showing that no broadening in the spectrum is observed in that plasma state. The bottom panels in Fig. 16 show the spectra in wave vector at two different times during the plasma evolution $t \omega_{d 0}=12$ and in the asymptotic time $t \omega_{d 0}=40$. The left bottom panel shows the plasma behavior in the intermediate regime at time $t \omega_{d 0}=12$ : Two peaks are occurring in the spectrum in toroidal number; we observe the initial excited mode and the appearance of a zonal flow structure. Such a structure grows strongly in that regime and becomes dominant leading to a global stabilization of the ITG instability.

It is found that in the negative-shear case with $s=-0.5$, zonal flows are enhanced and efficiently suppress the turbulent trapped ion heat transport. As the magnetic shear increases, the zonal flow level tends to decrease (for $s \geq 0.3$ ) and the ion transport increases. In plasma with higher magnetic shears $(s \geq 1)$, zonal flow component may become almost ignorable and streamer-type structures are then observed clearly. These streamer structures disappear for negative shears. Note also that the zonal flow is also visible in this Vlasov simulation, performed with a temperature gradient $\Delta \tau$ chosen well below the threshold $\Delta \tau_{s}$, which is a clear indication of the stabilization mechanism of the ITG instability by zonal flow. Indeed the marginal equilibrium plasma state is maintained by the formation of a zonal flow.

\section{CONCLUSION}

Trapped ion mode turbulence has been studied using a reduced Vlasov equation averaged over fast time scales, cyclotron, and bounce motion of trapped particles in the presence of a magnetic shear. Such instabilities are characterized by low frequencies of the order of the trapped particle precession frequency and radial scales of the order of several ion banana widths. TIMs are thus a simple prototype of kinetic instability induced by the resonance of trapped particles with waves or fluctuations through their precession motion which implies low toroidal numbers allowing fast global gyrokinetic simulations. Although such modes are predicted by Kadomtsev and Pogutse 40 years ago and are known to be subject to interchangelike instabilities, their hybrid fluid and kinetic aspects reveal new features; we may also meet in TEM turbulence, in particular, the property to generate a streamer-type turbulence.

A gyrokinetic Vlasov solver has been developed and implemented on a parallel computer using a semi-Lagrangian numerical scheme, the self-consistency being ensured by the quasineutrality condition. Such a code can simulate largescale structures by computing the wave-particle interactions from first principles. The accuracy afforded by our code allows us to obtain new insights and physical pictures of saturation mechanisms of the TIM turbulence and the resulting streamer-induced transport in the presence of magnetic shear. The nature of the final state (dominated by zonal flow or streamer) depends on the level of the threshold of the temperature (or density) gradient turbulence and of the strength of the magnetic shear. The magnetic shear is identified to play a key role in the saturation mechanism or large wavelength (TIM-like) structure selection. Spatial spectral analysis of TIM turbulence clearly display a condensation tendency of turbulent fluctuation energy to large wavelength (LSSS) structure component for high values of the magnetic shear and to zonal flow component to reverse or smaller magnetic shears. Such a process appears in a subcritical regime close to the threshold of the ITG instability where nonlinear parametriclike three-wave mode coupling is possible.

Many of the ideas that are developed in this paper can, in principle, be carried out for TEM turbulence. In addition a more comprehensive comparison of TIM- and TEM-driven streamers should include cases where the modes are strongly coupled. This is left for future work.

\section{ACKNOWLEDGMENTS}

The authors acknowledge the IDRIS (Institut du Développement et des Ressources pour l'Informatique Scientifique, CNRS, Orsay, France) for computer time allocation on their computers.

${ }^{1}$ G. Manfredi, C. M. Roach, and R. O. Dendy, Plasma Phys. Controlled Fusion 43, 825 (2001).

${ }^{2}$ A. Das, A. Sen, S. Mahajan, and P. Kaw, Phys. Plasmas 8, 5104 (2001).

${ }^{3}$ P. Manz, M. Ramisch, and V. Stroth, Phys. Rev. Lett. 103, 165004 (2009).

${ }^{4}$ P. H. Diamond, S. I. Itoh, and T. S. Hahm, Plasma Phys. Controlled Fusion 47, R35 (2005).

${ }^{5}$ A. Fujisawa, Nucl. Fusion 49, 013001 (2009). 
${ }^{6}$ J. Anderson, H. Nordman, R. Singh, and J. Weiland, Plasma Phys. Controlled Fusion 48, 651 (2006).

${ }^{7}$ L. Wang and T. S. Hahm, Phys. Plasmas 16, 082302 (2009).

${ }^{8}$ F. Merz and F. Jenko, Phys. Rev. Lett. 100, 035005 (2008).

${ }^{9}$ S. Champeaux and P. H. Diamond, Phys. Lett. A 288, 214 (2001).

${ }^{10}$ O. D. Gürcan and P. H. Diamond, Phys. Plasmas 11, 572 (2004).

${ }^{11}$ T. Hauff and F. Jenko, Phys. Plasmas 16, 102306 (2009).

${ }^{12}$ B. B. Kadomtsev and O. P. Pogutse, Nucl. Fusion 11, 67 (1971).

${ }^{13}$ D. J. Gambier and A. Samain, Nucl. Fusion 25, 283 (1985).

${ }^{14}$ A. Samain, Nucl. Fusion 12, 577 (1972).

${ }^{15}$ G. Depret, X. Garbet, P. Bertrand, and A. Ghizzo, Plasma Phys. Controlled Fusion 42, 949 (2000).

${ }^{16}$ X. Garbet, L. Laurent, F. Mourgues, J. P. Roubin, and A. Samain, J. Comput. Phys. 87, 249 (1990).

${ }^{17}$ X. Garbet, L. Laurent, and A. Samain, Phys. Plasmas 1, 850 (1994).

${ }^{18}$ A. J. Brizard and T. S. Hahm, Rev. Mod. Phys. 79, 421 (2007).

${ }^{19}$ J. R. Cary and A. J. Brizard, Rev. Mod. Phys. 81, 693 (2009).

${ }^{20}$ B. H. Fong and T. S. Hahm, Phys. Plasmas 6, 188 (1999).

${ }^{21}$ A. Hasegawa and K. Mima, Phys. Rev. Lett. 39, 205 (1977).

${ }^{22}$ D. Fyfe and D. Montgomery, Phys. Fluids 22, 246 (1979).

${ }^{23}$ R. H. Kraichnan, Phys. Fluids 10, 1417 (1967).

${ }^{24}$ P. W. Terry, R. Gatto, and D. A. Bauer, Phys. Rev. Lett. 89, 205001 (2002).

${ }^{25}$ T. S. Hahm and W. M. Tang, Phys. Plasmas 3, 242 (1996).

${ }^{26}$ A. Hasegawa and M. Wakatani, Phys. Rev. Lett. 50, 682 (1983).

${ }^{27}$ E. Kim and P. H. Diamond, Phys. Plasmas 10, 1698 (2003).
${ }^{28}$ I. Sandberg, H. Isliker, V. P. Pavlenko, K. Hizanidis, and L. Vlahos, Phys. Plasmas 12, 032503 (2005).

${ }^{29}$ D. Strintzi and F. Jenko, Phys. Plasmas 14, 042305 (2007).

${ }^{30}$ P. H. Diamond, S. Champeaux, M. Malkov, A. Das, I. Gruzinov, M. N. Rosenbluth, C. Holland, B. Wecht, A. I. Smolyakov, F. L. Hinton, Z. Lin, and T. S. Hahm, Nucl. Fusion 41, 1067 (2001).

${ }^{31} \mathrm{~J}$. Li and Y. Kishimoto, Phys. Plasmas 15, 112504 (2008).

${ }^{32}$ F. Jenko and B. D. Scott, Phys. Rev. Lett. 80, 4883 (1998).

${ }^{33}$ W. Deng and Z. Lin, Phys. Plasmas 16, 102503 (2009).

${ }^{34}$ C. Z. Cheng and G. Knorr, J. Comput. Phys. 22, 330 (1976).

${ }^{35}$ A. Ghizzo, B. Izrar, P. Bertrand, E. Fijalkow, M. R. Feix, and M. Shoucri, Phys. Fluids 31, 72 (1988).

${ }^{36}$ F. Huot, A. Ghizzo, P. Bertrand, E. Sonnendrücker, and O. Coulaud, J. Comput. Phys. 185, 512 (2003).

${ }^{37}$ A. N. Kolmogorov, J. Fluid Mech. 13, 82 (1962).

${ }^{38}$ P. W. Terry, Phys. Rev. Lett. 93, 235004 (2004).

${ }^{39}$ Y. Sarazin, V. Grandgirard, E. Fleurence, X. Garbet, Ph. Ghendrih, P. Bertrand, and G. Depret, Plasma Phys. Controlled Fusion 47, 1817 (2005).

${ }^{40}$ H. Xia and M. G. Shats, Phys. Rev. Lett. 91, 155001 (2003).

${ }^{41}$ X. Garbet, L. Laurent, A. Samain, and J. Chinardet, Nucl. Fusion 34, 963 (1994).

${ }^{42}$ T. S. Hahm, P. H. Diamond, Z. Lin, K. Itoh, and S. I. Itoh, Plasma Phys. Controlled Fusion 46, A323 (2004).

${ }^{43}$ Z. Lin and T. S. Hahm, Phys. Plasmas 11, 1099 (2004). 